Check for updates

Cite this: RSC Adv., 2017, 7, 22860

\title{
Regioselective thiolation of electron rich arenes and heterocycles in recyclable catalytic mediatt:
}

\author{
Dushyant Singh Raghuvanshi (D) and Narsingh Verma \\ A convenient and novel approach has been developed for the synthesis of unsymmetrical diaryl sulfides by \\ the reaction of sulfonyl hydrazides with phenols using a $[\mathrm{Bmim}][\mathrm{Br}]$ ionic liquid through the formation of \\ $\mathrm{C}-\mathrm{S}$ bonds. The reaction has further been extended to indole, $\beta$-naphthol and aromatic amine moieties. \\ This protocol offers a new, versatile and greener approach for thiolation of natural phenols \\ (monoterpenes)/aromatic phenols/ $\beta$-naphthols/aromatic amines and indoles without using any catalyst. \\ A broad range of functional groups were well tolerated in this reaction system.
}

Received 25th February 2017

Accepted 17th April 2017

DOI: $10.1039 / \mathrm{c} 7 \mathrm{ra02350b}$

rsc.li/rsc-advances

In the existing green chemistry scenario and increased

\section{Introduction}

The C-S bond formation is of utmost importance and has emerged as a key step in the syntheses of pharmaceuticals, natural products, and organic materials. ${ }^{1}$ But the current challenges in organic synthesis are to develop convenient, selective, and energy-efficient synthetic methods. In order to address these concerns, the exploration of more practical and environmentally benign synthetic methodologies is of contemporary interest. ${ }^{2}$ The $\mathrm{C}-\mathrm{S}$ bond formation reactions are not much explored because thiols are very prone to dimer formation (S-S coupling) leading to the formation of undesired disulfides and organic sulfur compounds act as metal deactivators due to their nature to bind with metals. ${ }^{3}$ In order to synthesize remarkably important diaryl sulfides, numerous catalytic reactions have been developed. ${ }^{4}$ In recent decades, scientists have paid much more attention to the development of new concepts for $\mathrm{C}-\mathrm{S}$ bond formation viz. direct oxidative coupling by selective functionalization of the $\mathrm{C}-\mathrm{H}$ bond. Such processes are endowed with high yields and impressive regio- and stereo-selectivity and are thus becoming a part of an ever-growing armory of useful synthetic tools available to organic chemists today. However, most of the present $\mathrm{C}-\mathrm{S}$ bond formation methods via $\mathrm{C}-\mathrm{H}$ bond activation rely on the use of transition metals such as copper, ${ }^{5}$ iron, ${ }^{6 \boldsymbol{a}}$ nickel, ${ }^{6 \boldsymbol{b}, \boldsymbol{c}}$ rhodium, ${ }^{7}$ palladium, ${ }^{8}$ etc. In these methods, different S-sources are used, for example, sulfonyl hydrazides, sodium sulfinates, diaryl disulfides, and arylthiols. However, these methods suffer from the use of toxic metal salts, harsh reaction conditions, and narrow substrate scopes.

Department of Medicinal Chemistry, CSIR-Central Institute of Medicinal and Aromatic Plants, P.O. CIMAP, Kukrail Road, Lucknow 226015, India. E-mail: dushyant.bhu@ gmail.com; dushyantsingh@cimap.res.in; Fax: +91 5222718695; Tel: +91 5222718588 $\dagger$ CIMAP Communication No. CIMAP/PUB/2016/83.

‡. Electronic supplementary information (ESI) available. See DOI: $10.1039 / \mathrm{c} 7 \mathrm{ra02350b}$ concern over environmental issues, many green approaches for $\mathrm{C}-\mathrm{S}$ bond formation have also been reported by the groups of Xiao, ${ }^{9}$ Yan, ${ }^{10}$ Jiang, ${ }^{11}$ Tian, ${ }^{12}$ and others. ${ }^{13}$ However, the generality of the existing reports is somewhat vitiated by the severe reaction conditions, and the catalysts and solvents used are not acceptable in the context of green synthesis. Moreover, the previous reports for the synthesis of diaryl sulfides have repeatedly used either iodine or iodine based catalysts (Scheme 1)..$^{\mathbf{9 1 0 , 1 2}}$ Which is also a major drawbacks of existing reports. Thus, the development of a new, efficient and green approach for the preparation of diaryl sulfides through C-S bond formation is highly desirable. The use of a benign and recyclable catalyst/solvent with high activity and selectivity is an interesting and rapidly growing area of synthetic chemistry. Owing to their green credentials, ionic liquids (ILs) have attracted considerable interest as environmentally benign reaction media, ${ }^{14-17}$ catalysts $^{17-19}$ and reagents. ${ }^{20}$

\section{Previous reports:}

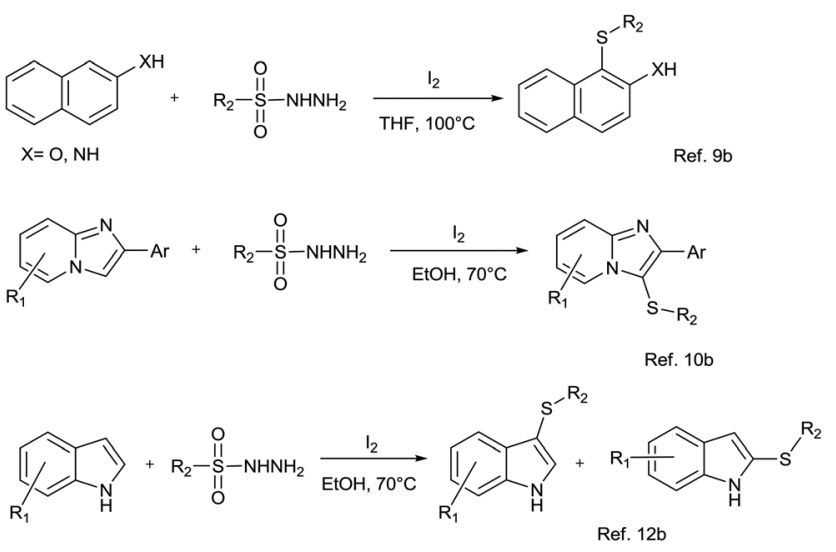

Scheme 1 lodine catalysed sulfenylation of aromatic systems. 


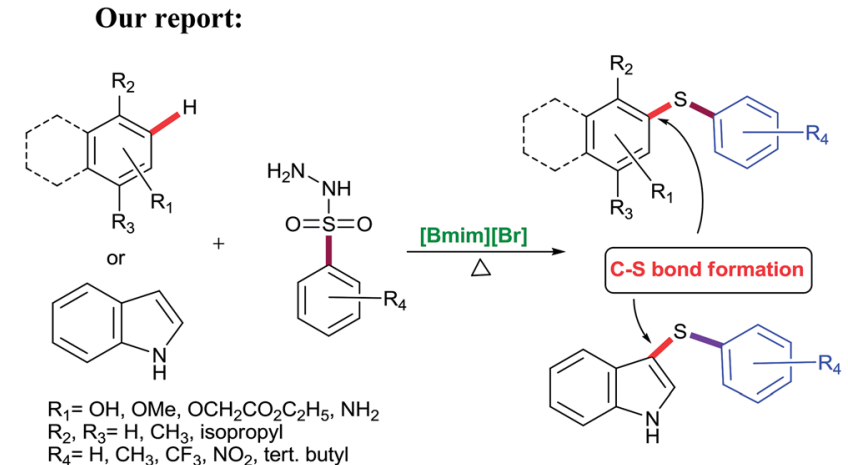

Scheme 2 C-S bond formation using $[\mathrm{Bmim}][\mathrm{Br}]$ ionic liquid.

Considering the significance of developing a green and highly efficient direct $\mathrm{C}-\mathrm{S}$ bond formation, and as a part of our ongoing research to design novel protocols including $\mathrm{C}-\mathrm{S}$ bond formation, ${ }^{21}$ we focused our attention towards the studies on the $[\mathrm{Bmim}][\mathrm{Br}]$ mediated $\mathrm{C}-\mathrm{S}$ bond formation of phenols (monoterpenes)/aromatic phenols/ $\beta$-naphthol/aromatic amines and indole (Scheme 2).

In this protocol, sulfonyl hydrazides were prime choice as a Ssource for $\mathrm{C}-\mathrm{S}$ bond formation due to their stability and versatile nature viz. as aryl sources, ${ }^{22}$ sulfonyl sources ${ }^{23}$ and reductants. ${ }^{24}$ To the best of our knowledge, this is a highly efficient and conceptually new methodology applied first time for C-S bond formation of phenols, $\beta$-naphthol, aromatic amines and indole using environmentally benign ionic liquid (Scheme 2).

\section{Results and discussion}

To look into the greener and efficient prospects of C-S bond formation, a model reaction using easily available substrates, tosylhydrazide and naturally isolated phenol i.e. thymol were investigated in detail by varying different parameters such as catalyst, temperature, additive, and solvent to develop the appropriate conditions (Table 1). At the outset, different greener and environmentally benign catalysts and reagents viz. TBAF, TBAI, $\mathrm{I}_{2} / \mathrm{SiO}_{2}, \mathrm{I}_{2} / \mathrm{Al}_{2} \mathrm{O}_{3}, \mathrm{I}_{2} / \mathrm{IR}-400,[\mathrm{Bmim}][\mathrm{Br}]$ were screened to determine their catalytic efficacy (Table 1, entries 1-6). We were astonished to see that only $[\mathrm{Bmim}][\mathrm{Br}]$ could bring the desired conversion (entry 6), and other catalysts did not provide the desired product rather gave undesired disulfides or no reaction (Table 1, entries 1-5). Encouraged by these results, our studies were directed to look at the prospective of other ionic liquids too viz. $[\mathrm{Bmim}][\mathrm{I}],[\mathrm{Bmim}][\mathrm{Cl}]$, $[\mathrm{Emim}][\mathrm{Cl}]$ and $[\mathrm{Emim}]\left[\mathrm{HSO}_{4}\right]$, but none of these could match the efficacy of $[\mathrm{Bmim}][\mathrm{Br}]$ which provided diaryl sulfide product 3a in $85 \%$ yield at $140{ }^{\circ} \mathrm{C}$ (entry 6). In order to screen the effect of temperature and time, the model reaction was undertaken under conventional conditions in pressure tube using $[\mathrm{Bmim}][\mathrm{Br}]$ as a reagent at varying temperatures and time periods. When the reaction temperature was elevated to $150{ }^{\circ} \mathrm{C}$ (entry 8), no remarkable change was observed in the product yield. At $130{ }^{\circ} \mathrm{C}$, low product yield was obtained (Table 1 , entry 7). The optimum conversion was achieved under catalystfree conditions at $140{ }^{\circ} \mathrm{C}$ in $5 \mathrm{~h}$ (Table 1). Tosylhydrazide (1.2 equiv.) and thymol (1.0 equiv.) were established as sufficient to obtain the maximum yield at $140^{\circ} \mathrm{C}$ in $[\mathrm{Bmim}][\mathrm{Br}]$ in $5 \mathrm{~h}$ (entry 6).

Table 1 Optimization of reaction conditions ${ }^{a}$

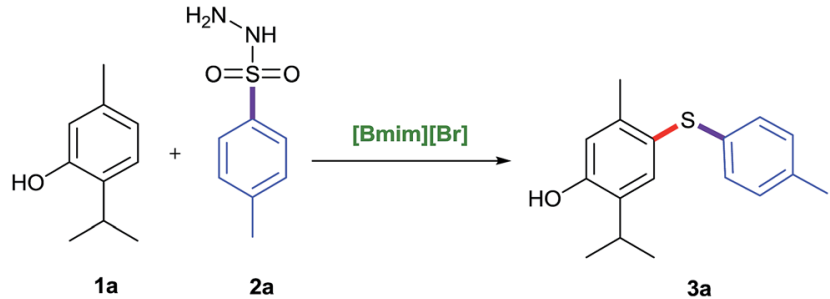

\begin{tabular}{|c|c|c|c|c|c|}
\hline S. no. & Solvent & Catalyst (mol\%) & Temp $\left({ }^{\circ} \mathrm{C}\right)$ & Time (h) & Yield $^{b}(\%)$ \\
\hline 1 & $\mathrm{H}_{2} \mathrm{O}$ & TBAF & 140 & 7 & $\mathrm{nr}^{c}$ \\
\hline 2 & $\mathrm{H}_{2} \mathrm{O}$ & TBAI & 140 & 7 & 30 \\
\hline 4 & $\mathrm{H}_{2} \mathrm{O}$ & $\mathrm{I}_{2} / \mathrm{Al}_{2} \mathrm{O}_{3}$ & 140 & 7 & $\mathrm{nr}$ \\
\hline 5 & $\mathrm{H}_{2} \mathrm{O}$ & $\mathrm{I}_{2} / \mathrm{IR} 400$ & 140 & 7 & Trace \\
\hline 6 & {$[\mathrm{Bmim}][\mathrm{Br}]$} & - & 140 & 5 & 85 \\
\hline 9 & {$[\mathrm{Bmim}][\mathrm{Br}]$} & - & 140 & 5 & $80^{d}$ \\
\hline 10 & {$[\mathrm{Bmim}][\mathrm{Br}]$} & - & 140 & 7 & 84 \\
\hline 11 & {$[\mathrm{Bmim}][\mathrm{I}]$} & - & 140 & 5 & 55 \\
\hline 12 & {$[\mathrm{Bmim}][\mathrm{Cl}]$} & - & 140 & 5 & 52 \\
\hline 13 & {$[\mathrm{Emim}][\mathrm{Cl}]$} & - & 140 & 5 & 48 \\
\hline 14 & {$[\mathrm{Emim}]\left[\mathrm{HSO}_{4}\right]$} & - & 140 & 5 & 10 \\
\hline
\end{tabular}

${ }^{a}$ Reaction conditions: 1a $(1 \mathrm{mmol}), \mathbf{2 a}(1.2 \mathrm{mmol}),[\mathrm{Bmim}][\mathrm{Br}](2 \mathrm{mmol}), 5 \mathrm{~h} .{ }^{b}$ Isolated yield based on $=\mathbf{1 a} .{ }^{c} \mathrm{nr}=$ no reaction. ${ }^{d}$ Reaction carried out using $2 \mathrm{a}$ (1.5 mmol). 
Table 2 Thiolation of substituted phenols and arylsulfonyl hydrazides ${ }^{a, b}$

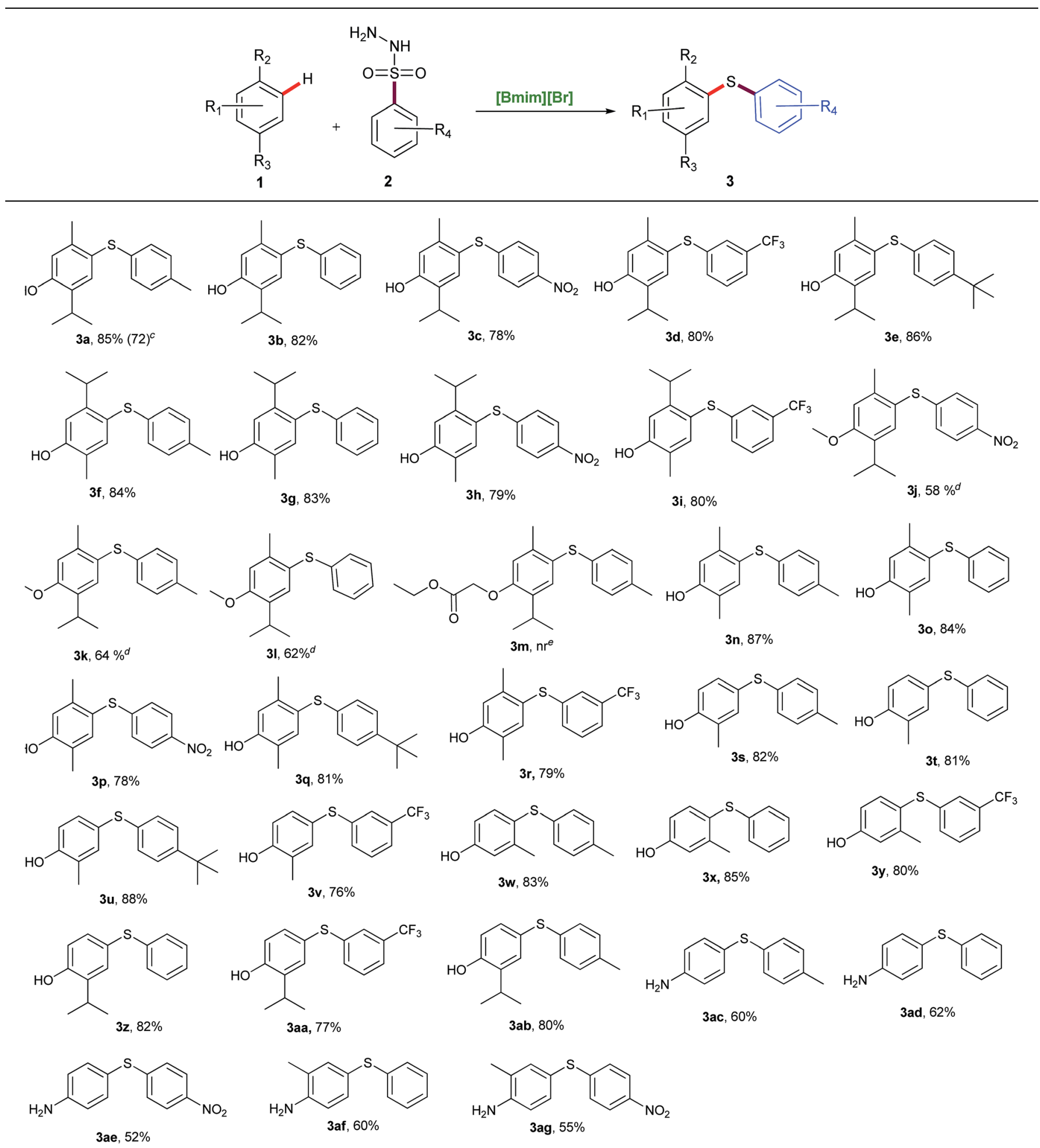

${ }^{a}$ Reaction conditions: $1(1 \mathrm{mmol}), 2(1.2 \mathrm{mmol}),[\mathrm{Bmim}][\mathrm{Br}](2 \mathrm{mmol}), 140{ }^{\circ} \mathrm{C}$ and $5 \mathrm{~h} .{ }^{b}$ Isolated yield based on phenols. ${ }^{c} \mathbf{1}(7 \mathrm{mmol}), 2(8.4 \mathrm{mmol})$, $[\mathrm{Bmim}][\mathrm{Br}](6 \mathrm{mmol}), 140{ }^{\circ} \mathrm{C}$ and $5 \mathrm{~h} .{ }^{d}$ Reaction carried out at $120^{\circ} \mathrm{C} .{ }^{e} \mathrm{nr}=$ no reaction. 
<smiles>Oc1ccc2ccccc2c1Sc1ccccc1Br</smiles><smiles>Oc1ccc2ccccc2c1</smiles>
$\begin{array}{ll}7 \mathrm{a}, \mathrm{R}_{1}=4-\mathrm{H} & \begin{array}{c}\text { Yields } \\ 85 \%\end{array} \\ 7 \mathrm{~b}, \mathrm{R}_{1}=4-\mathrm{tBu} & 80 \% \\ 7 \mathrm{c}, \mathrm{R}_{1}=4-\mathrm{CH}_{3} & 78 \%\end{array}$ $100^{\circ} \mathrm{C}$<smiles>NNS(=O)(=O)c1cccc(Br)c1</smiles><smiles>CC(C)(Br)Br</smiles><smiles>[R]1ccc(Sc2c[nH]c3ccccc23)cc1</smiles>
5

$\begin{array}{lcc} & \text { Yields } & \text { Time } \\ \mathbf{5 a}, \mathrm{R}_{1}=\mathbf{4}-\mathrm{H} & 65 \% & 5 \mathrm{hr} \\ \mathbf{5 b}, \mathrm{R}_{1}=3-\mathrm{CF}_{3} & 70 \% & 5 \mathrm{hr} \\ \mathbf{5 c}, \mathrm{R}_{1}=4-\mathrm{NO}_{2} & 82 \% & 5 \mathrm{hr} \\ \mathbf{5 d}, \mathrm{R}_{1}=4-\mathrm{CH}_{3} & 67 \% & 5 \mathrm{hr}\end{array}$

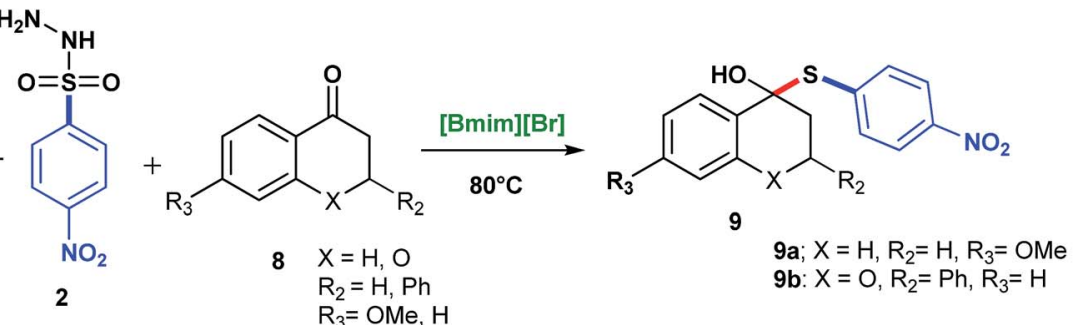

Scheme 3 C-S bond formation of indole, $\beta$-naphthol and ketone. Reaction conditions: 4, 6, 8 (1 mmol), 2 (1.2 mmol), [Bmim] [Br] (2 mmol), and $5 \mathrm{~h}$

Intrigued by these observations and with the stipulated reaction conditions in hand (Table 1, entry 6), the scope and versatility of this method was extended to the reaction of a variety of sulfonyl hydrazides viz. phenyl-, $p$-tolyl-, $p$-tertbutylphenyl-, $p$-nitrophenyl and 3-(trifluoromethyl) benzenesulfonyl hydrazide with different phenols like thymol, carvacrol, 2,5-dimethylphenol, $o$-cresol, $m$-cresol and thymol ether to afford a diverse range of unsymmetrical diaryl sulfides 3a-3ab in reasonably high yields (Table 2). The findings reveal that all sorts of arylsulfonyl hydrazides undergo reaction smoothly. It was worthwhile to mention that the nature of the substituent on the aromatic rings of the sulfonyl hydrazides did not significantly affect the yields of this reaction.

Sulfonyl hydrazides with electron-donating groups gave slightly higher yields than those with electron-withdrawing groups. When ether of thymol was subjected to the reaction, at the same set of optimized conditions, side product formation was more, so the reaction was again optimized for thymol ether and the best result was obtained at $120{ }^{\circ} \mathrm{C}$ in $5 \mathrm{~h}(3 \mathbf{j}-3 \mathbf{l})$. The reaction was also tried with ester derivative of thymol and tosylhydrazide (Table 2, entry $\mathbf{3 m}$ ) but it did not succeed.

$\beta$-Naphthol was also tried under the same set of optimized reaction conditions; which was going smoothly at $100{ }^{\circ} \mathrm{C}$ (Scheme 3). Encouraged by above results, the aromatic amines were also tried in place of phenols for $\mathrm{C}-\mathrm{S}$ bond formation and the reactions were going smoothly at the same set of optimized reaction conditions (Table 2, entry $\mathbf{3 a c}-\mathbf{3 a g}$ ). The substituent on benzene ring of aromatic amine and sulfonyl hydrazide did not significantly affect the product yields. Further, in order to ascertain the versatility of our reagent $[\mathrm{Bmim}][\mathrm{Br}]$ towards others functionality, we extended the scope of substrates and carried out the reactions of various arylsulfonyl hydrazides with indole under the aforesaid conditions (Scheme 3). It was found that indole gave reasonably good yields at $100{ }^{\circ} \mathrm{C}$ (Scheme 3$)$. To check the further feasibility of our methods, the aforemention conditions were screened for ketone functionality also. For this purpose, we performed a sulfenylation of substituted tetralone and chromanone with $p$-nitrobenzenesulfonyl hydrazide. When the above reaction was accomplished and products were analysed, we were confronted with some other spectral patterns not corroborating the formation of the expected product 10. The spectral analysis indicates the formation of product $\mathbf{9}$ (Scheme 3).

The recyclability of the ionic liquid was investigated with the same set of optimised reaction condition as a model reaction (Table 1, entry 6). Upon completion of the reaction, the product was isolated via standard work up procedure, while the aqueous layer containing $[\mathrm{Bmim}][\mathrm{Br}]$ was dried to remove water at $60^{\circ} \mathrm{C}$ under vacuum and then washed with diethyl ether. The recovered ionic liquid was reused for four to five times without significant loss of the activity. Results were represented in Fig. 1.

On the basis of existing literature and based on my previous work, ${ }^{21 c, 25}$ we propose a plausible mechanism for para-sulfenylation of thymol, which is outlined in Scheme 4. Initially, sulfonyl hydrazide (2) is transformed to form $\mathbf{D}$ in the presence of [Bmim] [Br]. In the next step electrophilic reaction of $\mathbf{D}$ with thymol (1) yields desired product 3 through the path a, or path $b$ (Scheme 4 ).

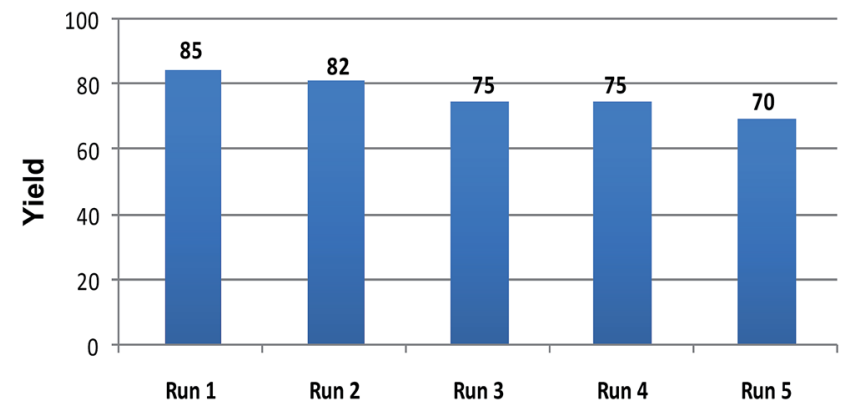

Fig. 1 Reusability of the ionic liquid. 


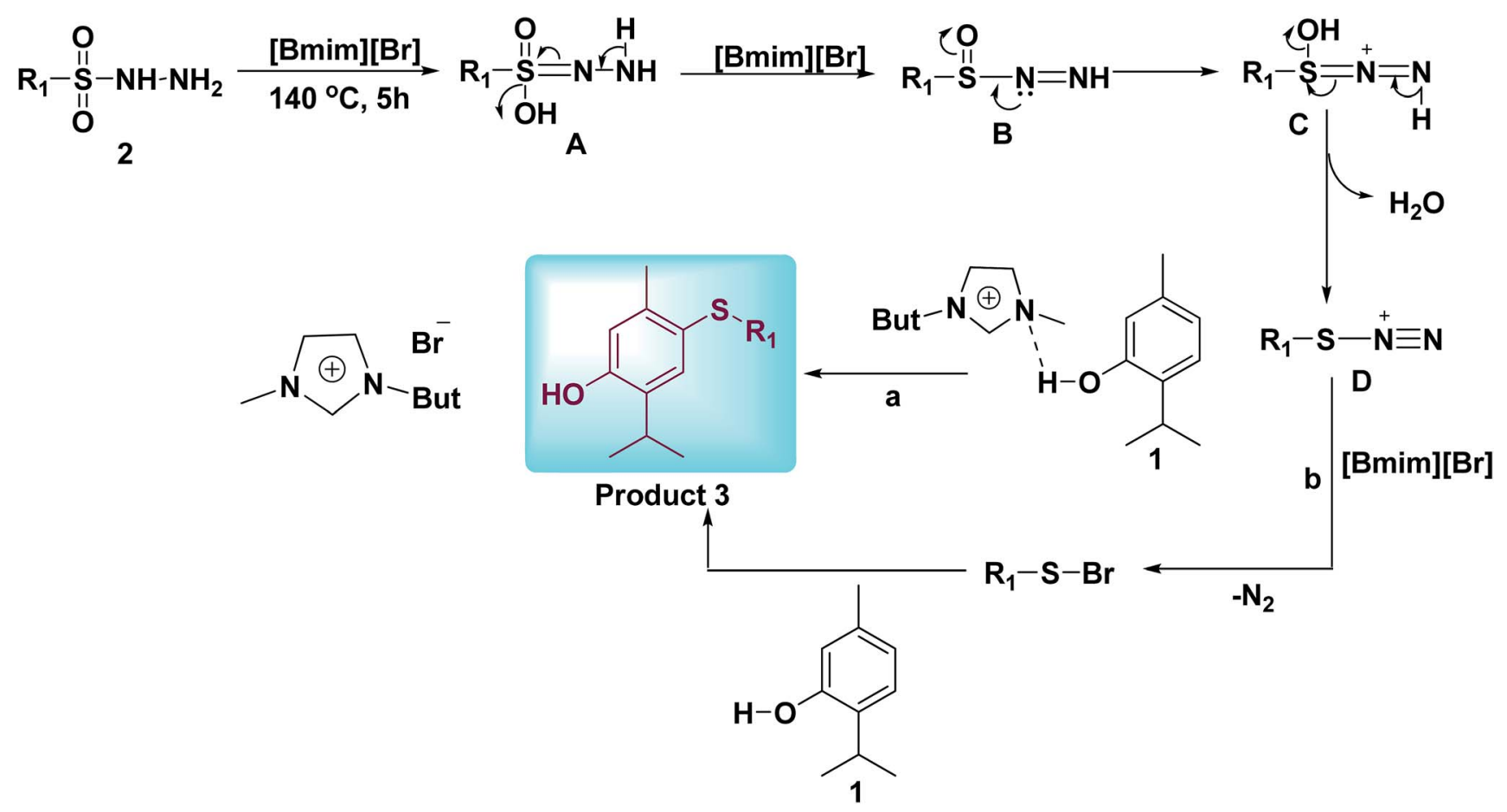

Scheme 4 Proposed mechanism.

\section{Conclusions}

We have developed a novel and efficient green protocol for regioselective thiolation of phenols/ $\beta$-naphthol, aromatic amines and indole without using any catalyst. The protocol embodies the first time use of [Bmim] [Br] ionic liquid, is efficient and versatile, and renders a new way of $\mathrm{C}-\mathrm{S}$ coupling. In this procedure, various substituents, such as alkyl, nitro, trifluoromethyl, and methoxy groups, are well tolerated. Due to high conversion, excellent regioselectivity, short reaction time and use of a recyclable ionic liquid, the process promises to be a practical and greener alternative. This method affords an efficient alternative approach for the synthesis of biologically important aryl sulphides.

\section{General experimental}

All the reactions were carried out in $15 \mathrm{~mL}$ Ace-pressure tube with constant stirring using Teflon-coated magnetic stirring bar. Column chromatography was performed using Merck silica gel (100-200 mesh). Thin layer chromatography (TLC) was performed using Merck $\mathrm{GF}_{254}$ plates (thickness $0.25 \mathrm{~mm}$ ). Visualization of spots on TLC plate was accomplished with UV light and by staining in $\mathrm{I}_{2}$ chamber. All the chemicals were procured from Sigma Aldrich and Alfa aessar.

\section{General procedure for the preparation of diaryl sulfides (C-S bond)}

A mixture of phenols 1 ( $1 \mathrm{mmol})$, arylsulfonyl hydrazides 2 (1.2 $\mathrm{mmol})$ and $[\mathrm{Bmim}][\mathrm{Br}](2 \mathrm{mmol})$, placed in a $15 \mathrm{~mL}$ Ace- pressure tube, was stirred at $140{ }^{\circ} \mathrm{C}$ for the stipulated period of time. After completion of the reaction (monitored through TLC), the mixture was cooled to room temperature and the reaction mixture was treated with EtOAc $(5 \mathrm{~mL})$ and water $(10$ $\mathrm{mL})$. This was extracted with ethyl acetate $(3 \times 10 \mathrm{~mL})$ and the combined organic phase was dried over $\mathrm{Na}_{2} \mathrm{SO}_{4}$, and the organic phase was concentrated under rotary vacuum evaporator and the crude product was purified by column chromatography using a mixture of ethyl acetate $/ n$-hexane as eluent to afford the desired unsymmetrical diaryl sulfides 3 .

\section{Spectral data of the compounds}

2-Isopropyl-5-methyl-4-(p-tolylthio)phenol (3a). Brown viscous liquid. ${ }^{1} \mathrm{H} \mathrm{NMR}\left(500 \mathrm{MHz}, \mathrm{CDCl}_{3}, \delta \mathrm{ppm}\right): 7.33$ (s, 1H, ArH), 7.03 $(\mathrm{d}, J=8.0 \mathrm{~Hz}, 2 \mathrm{H}, \mathrm{ArH}), 6.96(\mathrm{~d}, J=1.5 \mathrm{~Hz}, 2 \mathrm{H}, \operatorname{ArH}), 6.68(\mathrm{~s}, 1 \mathrm{H}$, $\mathrm{ArH}), 4.93$ (s, 1H, OH), 3.17-3.11 (m, 1H, CH), 2.28 (s, 3H, $\mathrm{CH}_{3}$ ), $2.27\left(\mathrm{~s}, 3 \mathrm{H}, \mathrm{CH}_{3}\right), 1.23\left(\mathrm{~d}, J=6.5 \mathrm{~Hz}, 6 \mathrm{H}, 2 \times \mathrm{CH}_{3}\right) .{ }^{13} \mathrm{C} \mathrm{NMR}$ (125 MHz, $\left.\mathrm{CDCl}_{3}, \delta \mathrm{ppm}\right): 153.49,140.78,134.92,134.29$, $133.04,129.64,127.06,123.14,117.65,26.89,22.52,20.89$, 20.31. EI-MS $\left(\mathrm{C}_{17} \mathrm{H}_{20} \mathrm{OS}\right): 272[\mathrm{M}], 273[\mathrm{M}+\mathrm{H}]^{+}$.

2-Isopropyl-5-methyl-4-(phenylthio)phenol (3b). Brown viscous liquid. ${ }^{1} \mathrm{H}$ NMR (500 MHz, $\left.\mathrm{CDCl}_{3}, \delta \mathrm{ppm}\right): 7.39$ (s, 1H, ArH), 7.28-7.21 (m, 2H, ArH), 7.13-7.05 (m, 1H, ArH), 7.05-7.04 (m, $2 \mathrm{H}, \mathrm{ArH}), 6.73(\mathrm{~s}, 1 \mathrm{H}, \mathrm{ArH}), 4.99(\mathrm{~s}, 1 \mathrm{H}, \mathrm{OH}), 3.21-3.15(\mathrm{~m}, 1 \mathrm{H}$, $\mathrm{CH}), 2.30\left(\mathrm{~s}, 3 \mathrm{H}, \mathrm{CH}_{3}\right), 1.27\left(\mathrm{~d}, J=6.5 \mathrm{~Hz}, 6 \mathrm{H}, 2 \times \mathrm{CH}_{3}\right) .{ }^{13} \mathrm{C}$ NMR (125 MHz, $\left.\mathrm{CDCl}_{3}, \delta \mathrm{ppm}\right):$ 153.77, 141.23, 138.85, 134.81, 133.21, 128.86, 126.43, 124.93, 122.30, 117.73, 26.88, 22.54, 22.50, 20.33. ESI-MS $\left(\mathrm{C}_{16} \mathrm{H}_{18} \mathrm{OS}\right): 259[\mathrm{M}+\mathrm{H}]^{+}$.

2-Isopropyl-5-methyl-4-((4-nitrophenyl)thio)phenol (3c). Yellow solid. Mp: $110-111^{\circ} \mathrm{C} .{ }^{1} \mathrm{H}$ NMR $\left(500 \mathrm{MHz}, \mathrm{CDCl}_{3}, \delta \mathrm{ppm}\right): 8.05-$ $8.02(\mathrm{~m}, 2 \mathrm{H}, \mathrm{ArH}), 7.37$ (s, 1H, ArH), 7.03-7.00 (m, 2H, ArH), 6.78 (s, 1H, ArH), $5.09(\mathrm{~s}, 1 \mathrm{H}, \mathrm{OH}), 3.21-3.15(\mathrm{~m}, 1 \mathrm{H}, \mathrm{CH}), 2.26(\mathrm{~s}, 3 \mathrm{H}$, 
$\mathrm{CH}_{3}$ ), 1.25 (d, $\left.J=7.0 \mathrm{~Hz}, 6 \mathrm{H}, 2 \times \mathrm{CH}_{3}\right) .{ }^{13} \mathrm{C} \mathrm{NMR}(125 \mathrm{MHz}$, $\left.\mathrm{CDCl}_{3}, \delta \mathrm{ppm}\right): 155.27,149.92,144.83,141.80,135.35,134.19$, 124.95, 124.0, 118.91, 118.18, 26.89, 22.49, 20.19. ESI-MS $\left(\mathrm{C}_{16} \mathrm{H}_{17} \mathrm{NO}_{3} \mathrm{~S}\right): 303[\mathrm{M}], 302[\mathrm{M}-\mathrm{H}]^{-}$.

2-Isopropyl-5-methyl-4-((3-(trifluoromethyl)phenyl)thio)phenol (3d). Colorless liquid. ${ }^{1} \mathrm{H} \mathrm{NMR}\left(500 \mathrm{MHz}, \mathrm{CDCl}_{3}, \delta \mathrm{ppm}\right): 7.37$ (s, 1H, ArH), 7.30-7.26 (m, 3H, ArH), 7.11 (d, $J=7.5 \mathrm{~Hz}, 1 \mathrm{H}, \operatorname{ArH})$, 6.74 (s, 1H, ArH), 5.13 (br s, 1H, OH), 3.18-3.14 (m, 1H, CH), $2.27\left(\mathrm{~s}, 3 \mathrm{H}, \mathrm{CH}_{3}\right), 1.24\left(\mathrm{~d}, J=7.0 \mathrm{~Hz}, 6 \mathrm{H}, 2 \times \mathrm{CH}_{3}\right) .{ }^{13} \mathrm{C} \mathrm{NMR}$ (125 MHz, $\left.\mathrm{CDCl}_{3}, \delta \mathrm{ppm}\right): 154.30,141.43,140.89,135.08$, 133.69, 131.64 (q), 129.13, 128.96, 124.97, 122.55, 121.48, 121.45, 120.76, 117.96, 26.86, 22.48, 20.25. EI-MS $\left(\mathrm{C}_{17} \mathrm{H}_{17} \mathrm{~F}_{3} \mathrm{OS}\right)$ : $326[\mathrm{M}], 327[\mathrm{M}+\mathrm{H}]^{+}$.

4-(4-tert-Butylphenylthio)-2-isopropyl-5-methylphenol

(3e). Colorless liquid. ${ }^{1} \mathrm{H}$ NMR (300 MHz, $\left.\mathrm{CDCl}_{3}, \delta \mathrm{ppm}\right): 7.28(\mathrm{~s}, 1 \mathrm{H}$, $\operatorname{ArH}), 7.17$ (d, $J=8.1 \mathrm{~Hz}, 2 \mathrm{H}, \operatorname{ArH}), 6.90$ (d, $J=8.4 \mathrm{~Hz}, 2 \mathrm{H}, \operatorname{ArH})$, 6.60 (s, 1H, ArH), 4.96 (br s, 1H, CH), 3.14-3.01 (m, 1H, CH), $2.22\left(\mathrm{~s}, 3 \mathrm{H}, \mathrm{CH}_{3}\right), 1.19\left(\mathrm{~s}, 9 \mathrm{H}, 3 \times \mathrm{CH}_{3}\right), 1.15(\mathrm{~d}, J=7.2 \mathrm{~Hz}, 6 \mathrm{H}, 2$ $\left.\times \mathrm{CH}_{3}\right) \cdot{ }^{13} \mathrm{C}$ NMR $\left(125 \mathrm{MHz}, \mathrm{CDCl}_{3}, \delta \mathrm{ppm}\right): 153.61,148.15$, 141.06, 135.11, 134.61, 133.06, 126.42, 125.90, 122.79, 117.62, $34.34,31.30,26.88,22.52$, 20.39. EI-MS $\left(\mathrm{C}_{20} \mathrm{H}_{26} \mathrm{OS}\right): 314$ [M], 315 $[\mathrm{M}+\mathrm{H}]^{+}$.

5-Isopropyl-2-methyl-4-(p-tolylthio)phenol (3f). Brown viscous liquid. ${ }^{1} \mathrm{H}$ NMR (500 MHz, $\left.\mathrm{CDCl}_{3}, \delta \mathrm{ppm}\right): 7.26(\mathrm{~s}, 1 \mathrm{H}, \mathrm{ArH}), 7.03$ (d, $J=8.0 \mathrm{~Hz}, 2 \mathrm{H}, \mathrm{ArH}), 6.98-6.95(\mathrm{~m}, 2 \mathrm{H}, \mathrm{ArH}), 6.79(\mathrm{~s}, 1 \mathrm{H}$, ArH), 5.12 (br s, 1H, OH), 3.53-3.48 (m, 1H, CH), $2.28(\mathrm{~s}, 3 \mathrm{H}$, $\left.\mathrm{CH}_{3}\right), 2.18\left(\mathrm{~s}, 3 \mathrm{H}, \mathrm{CH}_{3}\right), 1.14\left(\mathrm{~d}, J=6.5 \mathrm{~Hz}, 6 \mathrm{H}, 2 \times \mathrm{CH}_{3}\right) .{ }^{13} \mathrm{C}$ NMR (125 MHz, $\left.\mathrm{CDCl}_{3}, \delta \mathrm{ppm}\right):$ 154.92, 151.59, 138.61, 135.80, $134.94,129.60,127.28,122.30,121.96,112.88,30.62,23.76$, 20.89, 15.12. EI-MS $\left(\mathrm{C}_{17} \mathrm{H}_{20} \mathrm{OS}\right): 272[\mathrm{M}], 273[\mathrm{M}+\mathrm{H}]^{+}$.

5-Isopropyl-2-methyl-4-(phenylthio)phenol (3g). Brown viscous liquid. ${ }^{1} \mathrm{H}$ NMR (500 MHz, $\left.\mathrm{CDCl}_{3}, \delta \mathrm{ppm}\right): 7.30(\mathrm{~s}, 1 \mathrm{H}, \mathrm{ArH})$, 7.20-7.17 (m, 2H, ArH), 7.09-7.01 (m, 3H, ArH), 6.79 (s, 1H, ArH), 4.90 (br s, 1H, OH), 3.51-3.45 (m, 1H, CH), $2.19(\mathrm{~s}, 3 \mathrm{H}$, $\mathrm{CH}_{3}$ ), 1.13 (d, $\left.J=7.0 \mathrm{~Hz}, 6 \mathrm{H}, 2 \times \mathrm{CH}_{3}\right) .{ }^{13} \mathrm{C} \mathrm{NMR}(125 \mathrm{MHz}$, $\left.\mathrm{CDCl}_{3}, \delta \mathrm{ppm}\right): 155.20,152.11,139.77,139.21,128.78,126.59$, 124.90, 122.40, 121.08, 113.01, 30.70, 23.79, 15.11. ESI-MS $\left(\mathrm{C}_{16} \mathrm{H}_{18} \mathrm{OS}\right): 259[\mathrm{M}+\mathrm{H}]^{+}$.

5-Isopropyl-2-methyl-4-((4-nitrophenyl)thio)phenol (3h). Yellow viscous liquid. ${ }^{1} \mathrm{H}$ NMR (500 MHz, $\left.\mathrm{CDCl}_{3}, \delta \mathrm{ppm}\right): 8.03$ (dd, $J=$ 2.0, 7.0 Hz, 2H, ArH), 7.31 (s, 1H, ArH), 7.03 (dd, $J=2.0,7.0 \mathrm{~Hz}$, $2 \mathrm{H}, \operatorname{ArH}), 6.86(\mathrm{~s}, 1 \mathrm{H}, \operatorname{ArH}), 5.05(\mathrm{~s}, 1 \mathrm{H}, \mathrm{OH}), 3.37-3.31(\mathrm{~m}, 1 \mathrm{H}$, $\mathrm{CH}), 2.23\left(\mathrm{~s}, 3 \mathrm{H}, \mathrm{CH}_{3}\right), 1.14\left(\mathrm{~d}, J=7.0 \mathrm{~Hz}, 6 \mathrm{H}, 2 \times \mathrm{CH}_{3}\right) \cdot{ }^{13} \mathrm{C}$ NMR (125 MHz, $\left.\mathrm{CDCl}_{3}, \delta \mathrm{ppm}\right):$ 156.31, 152.88, 150.71, 144.80, 139.78, 125.07, 123.94, 123.27, 117.91, 113.55, 30.95, 23.83, 15.15. EI-MS $\left(\mathrm{C}_{16} \mathrm{H}_{17} \mathrm{NO}_{3} \mathrm{~S}\right): 303[\mathrm{M}], 304[\mathrm{M}+\mathrm{H}]^{+}$.

5-Isopropyl-2-methyl-4-((3-(trifluoromethyl)phenyl)thio)phenol (3i). Brown viscous liquid. ${ }^{1} \mathrm{H}$ NMR (500 $\left.\mathrm{MHz}, \mathrm{CDCl}_{3}, \delta \mathrm{ppm}\right)$ : 7.31-7.24 (m, 4H, ArH), 7.12 (d, J=7.0 Hz, 1H, ArH), 6.83 (s, 1H, $\mathrm{ArH}), 5.00$ (br s, 1H, OH), 3.43-3.38 (m, 1H, CH), $2.21(\mathrm{~s}, 3 \mathrm{H}$, $\mathrm{CH}_{3}$ ), 1.13 (d, $\left.J=7.0 \mathrm{~Hz}, 6 \mathrm{H}, 2 \times \mathrm{CH}_{3}\right) .{ }^{13} \mathrm{C}$ NMR $(125 \mathrm{MHz}$, $\left.\mathrm{CDCl}_{3}, \delta \mathrm{ppm}\right): 155.78,152.55,141.74,139.53,131.56$ (q), 129.07, 129.0, 124.98, 122.85, 122.54, 121.44, 121.40, 119.32, 113.34, 30.81, 23.78, 15.12. EI-MS $\left(\mathrm{C}_{17} \mathrm{H}_{17} \mathrm{~F}_{3} \mathrm{OS}\right): 326[\mathrm{M}], 327$ [M $+\mathrm{H}]^{+}$.

(5-Isopropyl-4-methoxy-2-methylphenyl)(4-nitrophenyl)sulfane (3j). Yellow solid. Mp: 104-105 ${ }^{\circ} \mathrm{C} .{ }^{1} \mathrm{H}$ NMR (500 MHz, $\mathrm{CDCl}_{3}$, $\delta$ ppm): 8.04 (d, $J=8.5 \mathrm{~Hz}, 2 \mathrm{H}, \mathrm{ArH}), 7.36$ (s, 1H, ArH), 7.02 (d, $J$ $=8.5 \mathrm{~Hz}, 2 \mathrm{H}, \mathrm{ArH}), 6.84(\mathrm{~s}, 1 \mathrm{H}, \mathrm{ArH}), 3.88\left(\mathrm{~s}, 3 \mathrm{H}, \mathrm{OCH}_{3}\right), 3.30-$ $3.25(\mathrm{~m}, 1 \mathrm{H}, \mathrm{CH}), 2.33\left(\mathrm{~s}, 3 \mathrm{H}, \mathrm{CH}_{3}\right), 1.20(\mathrm{~d}, J=7.0 \mathrm{~Hz}, 6 \mathrm{H}, 2 \times$ $\left.\mathrm{CH}_{3}\right) .{ }^{13} \mathrm{C}$ NMR (125 MHz, $\left.\mathrm{CDCl}_{3}, \delta \mathrm{ppm}\right):$ 158.61, 149.92, $144.81,141.78,136.65,134.65,124.97,124.0,118.65,113.10$, 55.48, 26.59, 22.57, 20.74. EI-MS $\left(\mathrm{C}_{17} \mathrm{H}_{19} \mathrm{NO}_{3} \mathrm{~S}\right): 317[\mathrm{M}], 318[\mathrm{M}$ $+\mathrm{H}]^{+}$.

(5-Isopropyl-4-methoxy-2-methylphenyl)(p-tolyl)sulfane (3k). Colorless viscous liquid. ${ }^{1} \mathrm{H}$ NMR (500 MHz, DMSO-d ${ }_{6}, \delta$ ppm): 7.34 (s, 1H, ArH), 7.04 (d, $J=8.0 \mathrm{~Hz}, 2 \mathrm{H}, \mathrm{ArH}), 6.97$ (m, 2H, ArH), $6.78(\mathrm{~s}, 1 \mathrm{H}, \mathrm{ArH}), 3.85\left(\mathrm{~s}, 3 \mathrm{H}, \mathrm{OCH}_{3}\right), 3.29-3.23(\mathrm{~m}, 1 \mathrm{H}, \mathrm{CH}), 2.36$ (s, $\left.3 \mathrm{H}, \mathrm{CH}_{3}\right), 2.29\left(\mathrm{~s}, 3 \mathrm{H}, \mathrm{CH}_{3}\right), 1.19\left(\mathrm{~d}, J=6.5 \mathrm{~Hz}, 6 \mathrm{H}, 2 \times \mathrm{CH}_{3}\right)$. ${ }^{13} \mathrm{C}$ NMR (125 MHz, $\left.\mathrm{CDCl}_{3}, \delta \mathrm{ppm}\right): 157.42,140.52,135.70$, 135.06, 134.86, 133.67, 129.63, 127.13, 122.60, 112.79, 55.44, 26.58, 22.63, 20.84. EI-MS $\left(\mathrm{C}_{18} \mathrm{H}_{22} \mathrm{OS}\right): 286[\mathrm{M}], 287[\mathrm{M}+\mathrm{H}]^{+}$.

(5-Isopropyl-4-methoxy-2-methylphenyl)(phenyl)sulfane (3l). Yellow viscous liquid. ${ }^{1} \mathrm{H}$ NMR $\left(500 \mathrm{MHz}, \mathrm{CDCl}_{3}, \delta \mathrm{ppm}\right): 7.35$ (s, 1H, ArH), 7.21-7.18 (m, 2H, ArH), 7.10-7.01 (m, 3H, ArH), $6.79(\mathrm{~s}, 1 \mathrm{H}, \mathrm{ArH}), 3.85\left(\mathrm{~s}, 3 \mathrm{H}, \mathrm{OCH}_{3}\right), 3.28-3.23(\mathrm{~m}, 1 \mathrm{H}, \mathrm{CH}), 2.35$ $\left(\mathrm{s}, 3 \mathrm{H}, \mathrm{CH}_{3}\right), 1.19\left(\mathrm{~d}, J=7.0 \mathrm{~Hz}, 6 \mathrm{H}, 2 \times \mathrm{CH}_{3}\right) \cdot{ }^{13} \mathrm{C} \mathrm{NMR}(125$ $\left.\mathrm{MHz}, \mathrm{CDCl}_{3}, \delta \mathrm{ppm}\right): 157.64,141.01,138.97,135.81,134.16$, 128.82 , 126.45, 124.86, 121.69, 112.79, 55.43, 26.55, 22.62, 20.86. EI-MS $\left(\mathrm{C}_{17} \mathrm{H}_{20} \mathrm{OS}\right): 272[\mathrm{M}], 273[\mathrm{M}+\mathrm{H}]^{+}$.

Ethyl 2-(2-isopropyl-5-methyl-4-(p-tolylthio)phenoxy)acetate (3m). No reaction.

2,5-Dimethyl-4-(p-tolylthio)phenol (3n). White solid. Mp: 96$97{ }^{\circ} \mathrm{C} .{ }^{1} \mathrm{H}$ NMR $\left(500 \mathrm{MHz}, \mathrm{CDCl}_{3}, \delta \mathrm{ppm}\right): 7.25$ (s, 1H, ArH), 7.04 $(\mathrm{d}, J=8.0 \mathrm{~Hz}, 2 \mathrm{H}, \mathrm{ArH}), 6.98(\mathrm{~d}, J=8.5 \mathrm{~Hz}, 2 \mathrm{H}, \mathrm{ArH}), 6.70(\mathrm{~s}, 1 \mathrm{H}$, $\mathrm{ArH}), 4.84(\mathrm{br} \mathrm{s}, 1 \mathrm{H}, \mathrm{OH}), 2.28\left(\mathrm{~s}, 6 \mathrm{H}, 2 \times \mathrm{CH}_{3}\right), 2.20(\mathrm{~s}, 3 \mathrm{H}$, $\left.\mathrm{CH}_{3}\right) .{ }^{13} \mathrm{C}$ NMR (125 MHz, $\left.\mathrm{CDCl}_{3}, \delta \mathrm{ppm}\right):$ 154.41, 141.03, 138.18, 135.11, 134.76, 129.69, 127.44, 123.16, 122.27, 117.24, 20.93, 20.40, 15.13. EI-MS $\left(\mathrm{C}_{15} \mathrm{H}_{16} \mathrm{OS}\right): 244[\mathrm{M}], 245[\mathrm{M}+\mathrm{H}]^{+}$.

2,5-Dimethyl-4-(phenylthio)phenol (3o). Colorless liquid. ${ }^{1} \mathrm{H}$ NMR (500 MHz, $\left.\mathrm{CDCl}_{3}, \delta \mathrm{ppm}\right): 7.30$ (s, 1H, ArH), 7.21-7.18 (m, 2H, ArH), 7.09-7.01 (m, 3H, ArH), 6.73 (s, 1H, ArH), 4.91 (s, 1H, $\mathrm{OH}), 2.28\left(\mathrm{~s}, 3 \mathrm{H}, \mathrm{CH}_{3}\right), 2.20\left(\mathrm{~s}, 3 \mathrm{H}, \mathrm{CH}_{3}\right) .{ }^{13} \mathrm{C} \mathrm{NMR}(125 \mathrm{MHz}$, $\left.\mathrm{CDCl}_{3}, \delta \mathrm{ppm}\right): 154.80,141.58,138.85,138.79,129.23,128.85$, $127.83,126.62$, 124.99, 122.42, 122.12, 117.30, 20.40, 15.11. ESIMS $\left(\mathrm{C}_{14} \mathrm{H}_{14} \mathrm{OS}\right): 229[\mathrm{M}-\mathrm{H}]^{-}$.

2,5-Dimethyl-4-((4-nitrophenyl)thio)phenol (3p). Yellow solid. Mp: $156-157{ }^{\circ} \mathrm{C} .{ }^{1} \mathrm{H}$ NMR (500 MHz, $\left.\mathrm{CDCl}_{3}, \delta \mathrm{ppm}\right): 8.04-8.01$ (m, 2H, ArH), 7.33 (s, 1H, $\left.\mathrm{CH}_{3}\right), 7.02-7.0(\mathrm{~m}, 2 \mathrm{H}, \mathrm{ArH}), 6.80(\mathrm{~s}$, $1 \mathrm{H}, \mathrm{ArH}), 5.13$ (s, 1H, OH), 2.27 (s, 3H, $\left.\mathrm{CH}_{3}\right), 2.23$ (s, 3H, $\left.\mathrm{CH}_{3}\right)$. ${ }^{13} \mathrm{C}$ NMR (125 MHz, $\left.\mathrm{CDCl}_{3}, \delta \mathrm{ppm}\right): 155.89,149.73,144.85$, 142.34, 139.56, 125.05, 124.01, 123.25, 118.99, 117.73, 20.27, 15.13. ESI-MS $\left(\mathrm{C}_{14} \mathrm{H}_{13} \mathrm{NO}_{3} \mathrm{~S}\right): 274[\mathrm{M}-\mathrm{H}]^{-}$.

4-((4-(tert-Butyl)phenyl)thio)-2,5-dimethylphenol (3q). Colorless liquid. ${ }^{1} \mathrm{H} \mathrm{NMR}\left(500 \mathrm{MHz}, \mathrm{CDCl}_{3}, \delta \mathrm{ppm}\right): 7.29$ (s, 1H, ArH), 7.24 (dd, $J=2.0,6.5 \mathrm{~Hz}, 2 \mathrm{H}, \mathrm{ArH}), 6.99$ (d, $J=0.5 \mathrm{~Hz}, 1 \mathrm{H}, \mathrm{ArH}), 6.97$ (s, 1H, ArH), $6.72(\mathrm{~s}, 1 \mathrm{H}, \operatorname{ArH}), 5.06$ (br s, 1H, OH), $2.31(\mathrm{~s}, 3 \mathrm{H}$, $\left.\mathrm{CH}_{3}\right), 2.19\left(\mathrm{~s}, 3 \mathrm{H}, \mathrm{CH}_{3}\right), 1.28\left(\mathrm{~s}, 9 \mathrm{H}, 3 \times \mathrm{CH}_{3}\right) \cdot{ }^{13} \mathrm{C}$ NMR (125 $\left.\mathrm{MHz}, \mathrm{CDCl}_{3}, \delta \mathrm{ppm}\right): 154.60,148.26,141.34,138.57,135.03$, $126.71,125.93,122.69,122.35,117.21,34.35,31.31,20.48$, 15.15. ESI-MS $\left(\mathrm{C}_{18} \mathrm{H}_{22} \mathrm{OS}\right): 285[\mathrm{M}-\mathrm{H}]^{-}$.

2,5-Dimethyl-4-(3-(trifluoromethyl)phenylthio)phenol

(3r). White solid. Mp: $98-99{ }^{\circ} \mathrm{C} .{ }^{1} \mathrm{H}$ NMR $\left(500 \mathrm{MHz}, \mathrm{CDCl}_{3}, \delta \mathrm{ppm}\right)$ : 7.32-7.26 (m, 4H, ArH), 7.10 (d, $J=7.5 \mathrm{~Hz}, 1 \mathrm{H}, \mathrm{ArH}), 6.76$ (s, 1H, 
$\mathrm{ArH}), 4.89(\mathrm{~s}, 1 \mathrm{H}, \mathrm{OH}), 2.27\left(\mathrm{~s}, 3 \mathrm{H}, \mathrm{CH}_{3}\right), 2.22\left(\mathrm{~s}, 3 \mathrm{H}, \mathrm{CH}_{3}\right) .{ }^{13} \mathrm{C}$ NMR (125 MHz, $\left.\mathrm{CDCl}_{3}, \delta \mathrm{ppm}\right):$ 155.31, 141.95, 140.77, 139.26, 131.37 (q), 129.15, 129.04, 122.79, 122.63, 121.54, 121.51, 120.52, 117.53, 20.33, 15.10. EI-MS $\left(\mathrm{C}_{15} \mathrm{H}_{13} \mathrm{~F}_{3} \mathrm{OS}\right): 298$ [M], 299 $[\mathrm{M}+\mathrm{H}]^{+}$.

2-Methyl-4-(p-tolylthio)phenol (3s). Colorless viscous liquid. ${ }^{1} \mathrm{H}$ NMR (500 MHz, $\mathrm{CDCl}_{3}, \delta \mathrm{ppm}$ ): 7.23 (s, 1H, ArH), 7.22-7.11 $(\mathrm{m}, 3 \mathrm{H}, \mathrm{ArH}), 7.07$ (d, $J=8.5 \mathrm{~Hz}, 2 \mathrm{H}, \mathrm{ArH}), 6.74(\mathrm{~d}, J=8.0 \mathrm{~Hz}$, $1 \mathrm{H}, \mathrm{ArH}), 4.82(\mathrm{~s}, 1 \mathrm{H}, \mathrm{OH}), 2.30\left(\mathrm{~s}, 3 \mathrm{H}, \mathrm{CH}_{3}\right), 2.21\left(\mathrm{~s}, 3 \mathrm{H}, \mathrm{CH}_{3}\right)$. ${ }^{13} \mathrm{C}$ NMR (125 MHz, $\left.\mathrm{CDCl}_{3}, \delta \mathrm{ppm}\right): 153.83,136.02,135.84$, 134.51, 132.17, 129.75, 129.23, 125.38, 125.01, 115.82, 20.99, 15.66. ESI-MS $\left(\mathrm{C}_{14} \mathrm{H}_{14} \mathrm{OS}\right): 229[\mathrm{M}-\mathrm{H}]^{-}$.

2-Methyl-4-(phenylthio)phenol (3t). Yellow viscous liquid. ${ }^{1} \mathrm{H}$ NMR (500 MHz, $\mathrm{CDCl}_{3}, \delta \mathrm{ppm}$ ): 7.28-7.20 (m, 4H, ArH), 7.17$7.11(\mathrm{~m}, 3 \mathrm{H}, \mathrm{ArH}), 6.77$ (d, $J=8.5 \mathrm{~Hz}, 1 \mathrm{H}, \mathrm{ArH}), 4.90$ (s, 1H, OH), $2.23\left(\mathrm{~s}, 3 \mathrm{H}, \mathrm{CH}_{3}\right) .{ }^{13} \mathrm{C} \mathrm{NMR}\left(125 \mathrm{MHz}, \mathrm{CDCl}_{3}, \delta \mathrm{ppm}\right): 154.25$, 138.75, 136.78, 133.13, 128.90, 128.12, 125.68, 125.19, 124.10, 115.94, 16.65. ESI-MS $\left(\mathrm{C}_{13} \mathrm{H}_{12} \mathrm{OS}\right): 215[\mathrm{M}-\mathrm{H}]^{-}$.

4-((4-(tert-Butyl)phenyl)thio)-2-methylphenol (3u). Colorless viscous liquid. ${ }^{1} \mathrm{H} \mathrm{NMR}\left(500 \mathrm{MHz}, \mathrm{CDCl}_{3}, \delta \mathrm{ppm}\right): 7.28-7.25(\mathrm{~m}$, $3 \mathrm{H}, \mathrm{ArH}), 7.21$ (dd, $J=2.0,8.5 \mathrm{~Hz}, 1 \mathrm{H}, \operatorname{ArH}), 7.14(\mathrm{~d}, J=8.5 \mathrm{~Hz}$, $2 \mathrm{H}, \mathrm{ArH}), 6.75$ (d, $J=8.5 \mathrm{~Hz}, 1 \mathrm{H}, \mathrm{ArH}), 4.98$ (br s, 1H, OH), 2.23 $\left(\mathrm{s}, 3 \mathrm{H}, \mathrm{CH}_{3}\right), 1.29\left(\mathrm{~s}, 9 \mathrm{H}, 3 \times \mathrm{CH}_{3}\right) \cdot{ }^{13} \mathrm{C} \mathrm{NMR}\left(125 \mathrm{MHz}, \mathrm{CDCl}_{3}\right.$, $\delta$ ppm): 154.03, 149.10, 136.37, 134.85, 132.69, 128.36, 126.02, $125.10,124.77,115.88,34.43,31.30,15.69$. EI-MS $\left(\mathrm{C}_{17} \mathrm{H}_{20} \mathrm{OS}\right)$ : $272[\mathrm{M}], 273[\mathrm{M}+\mathrm{H}]^{+}$.

2-Methyl-4-(3-(trifluoromethyl)phenylthio)phenol (3v). Yellow viscous liquid. ${ }^{1} \mathrm{H} \mathrm{NMR}\left(500 \mathrm{MHz}, \mathrm{CDCl}_{3}, \delta \mathrm{ppm}\right): 7.37-7.29(\mathrm{~m}$, $4 \mathrm{H}, \operatorname{ArH}), 7.25-7.23$ (m, 2H, ArH), 6.81 (d, $J=8.5 \mathrm{~Hz}, 1 \mathrm{H}, \operatorname{ArH})$, 5.00 (s, $1 \mathrm{H}, \mathrm{OH}), 2.25$ (s, $\left.3 \mathrm{H}, \mathrm{CH}_{3}\right) .{ }^{13} \mathrm{C} \mathrm{NMR}\left(125 \mathrm{MHz} \mathrm{CDCl}_{3}\right.$, $\delta$ ppm): 154.88, 140.97, 137.49, 133.92, 131.39, 131.13, 130.31, 129.17, 125.61, 123.80, 123.77, 122.21, 122.08, 122.05, 116.25, 15.65. EI-MS $\left(\mathrm{C}_{14} \mathrm{H}_{11} \mathrm{~F}_{3} \mathrm{OS}\right): 284[\mathrm{M}], 285[\mathrm{M}+\mathrm{H}]^{+}$.

3-Methyl-4-(p-tolylthio)phenol (3w). Brown viscous liquid. ${ }^{1} \mathrm{H}$ NMR (500 MHz, $\left.\mathrm{CDCl}_{3}, \delta \mathrm{ppm}\right): 7.33$ (d, $J=8.0 \mathrm{~Hz}, 1 \mathrm{H}, \operatorname{ArH}$ ), 7.05 (d, $J=8.0 \mathrm{~Hz}, 2 \mathrm{H}, \operatorname{ArH}), 7.00(\mathrm{~m}, 2 \mathrm{H}, \operatorname{ArH}), 6.77$ (d, $J=$ $2.5 \mathrm{~Hz}, 1 \mathrm{H}, \mathrm{ArH}$ ), 6.67 (dd, $J=3.0,8.5 \mathrm{~Hz}, 1 \mathrm{H}, \mathrm{ArH}$ ), 5.04 (br s, $1 \mathrm{H}, \mathrm{OH}), 2.32\left(\mathrm{~s}, 3 \mathrm{H}, \mathrm{CH}_{3}\right), 2.29\left(\mathrm{~s}, 3 \mathrm{H}, \mathrm{CH}_{3}\right) .{ }^{13} \mathrm{C}$ NMR (125 $\left.\mathrm{MHz}, \mathrm{CDCl}_{3}, \delta \mathrm{ppm}\right): 156.04,143.46,136.70,135.40,134.30$, $129.75,127.88,123.99,117.67,113.83,20.93$, 20.88. EI-MS $\left(\mathrm{C}_{14} \mathrm{H}_{14} \mathrm{OS}\right): 230[\mathrm{M}], 231[\mathrm{M}+\mathrm{H}]^{+}$.

3-Methyl-4-(phenylthio)phenol (3x). Colorless viscous liquid. ${ }^{1} \mathrm{H}$ NMR (500 MHz, $\left.\mathrm{CDCl}_{3}, \delta \mathrm{ppm}\right): 7.39$ (d, $\left.J=8.0 \mathrm{~Hz}, 1 \mathrm{H}, \mathrm{ArH}\right)$, 7.22-7.19 (m, 2H, ArH), 7.11-7.08 (m, 1H, ArH), 7.04-7.03 (m, $2 \mathrm{H}, \operatorname{ArH}), 6.80(\mathrm{~d}, J=2.5 \mathrm{~Hz}, 1 \mathrm{H}, \operatorname{ArH}), 6.69(\mathrm{dd}, J=3.0 \mathrm{~Hz}$, $8.0 \mathrm{~Hz}, 1 \mathrm{H}, \mathrm{ArH}), 4.92$ (br s, 1H, OH), $2.32\left(\mathrm{~s}, 3 \mathrm{H}, \mathrm{CH}_{3}\right) .{ }^{13} \mathrm{C} \mathrm{NMR}$ (125 MHz, $\left.\mathrm{CDCl}_{3}, \delta \mathrm{ppm}\right): 156.42,144.17,138.40,137.56$, 128.91, 126.95, 125.21, 122.92, 117.77, 113.93, 20.91. ESI-MS $\left(\mathrm{C}_{13} \mathrm{H}_{12} \mathrm{OS}\right): 239[\mathrm{M}+\mathrm{Na}]^{+}$.

3-Methyl-4-(3-(trifluoromethyl)phenylthio)phenol (3y). Brown viscous liquid. ${ }^{1} \mathrm{H}$ NMR (500 $\left.\mathrm{MHz}, \mathrm{CDCl}_{3}, \delta \mathrm{ppm}\right): 7.43$ (d, $J=$ $8.0 \mathrm{~Hz}, 1 \mathrm{H}, \mathrm{ArH}), 7.33-7.26$ (m, 3H, ArH), 7.12 (d, $J=7.5 \mathrm{~Hz}, 1 \mathrm{H}$, $\operatorname{ArH}), 6.83(\mathrm{~d}, J=2.5 \mathrm{~Hz}, 1 \mathrm{H}, \operatorname{ArH}), 6.73(\mathrm{dd}, J=2.5,8.0 \mathrm{~Hz}, 1 \mathrm{H}$, $\mathrm{ArH}), 5.11(\mathrm{~s}, 1 \mathrm{H}, \mathrm{OH}), 2.32\left(\mathrm{~s}, 3 \mathrm{H}, \mathrm{CH}_{3}\right) .{ }^{13} \mathrm{C} \mathrm{NMR}(125 \mathrm{MHz}$, $\left.\mathrm{CDCl}_{3}, \delta \mathrm{ppm}\right): 156.96,144.66,140.46,138.20,131.70$ (q), 129.27, 124.93, 122.84, 121.70, 121.73, 121.20, 118.05, 114.28, 20.85. EI-MS $\left(\mathrm{C}_{14} \mathrm{H}_{11} \mathrm{~F}_{3} \mathrm{OS}\right): 284[\mathrm{M}], 285[\mathrm{M}+\mathrm{H}]^{+}$.
2-Isopropyl-4-(phenylthio)phenol (3z). Brown viscous liquid. ${ }^{1} \mathrm{H}$ NMR (500 MHz, $\left.\mathrm{CDCl}_{3}, \delta \mathrm{ppm}\right): 7.34$ (d, $\left.J=2.0 \mathrm{~Hz}, 1 \mathrm{H}, \mathrm{ArH}\right)$, 7.24-7.11 (m, 6H, ArH), 6.74 (d, J=8.0 Hz, 1H, ArH), 4.93 (s, 1H, $\mathrm{OH}), 3.21-3.16(\mathrm{~m}, 1 \mathrm{H}, \mathrm{CH}), 1.24\left(\mathrm{~d}, J=6.5 \mathrm{~Hz}, 6 \mathrm{H}, 2 \times \mathrm{CH}_{3}\right)$. ${ }^{13} \mathrm{C}$ NMR (125 MHz, $\left.\mathrm{CDCl}_{3}, \delta \mathrm{ppm}\right): 153.20,138.85,135.79$, $132.68,132.62$, 128.90, 127.87, 125.59, 124.22, 116.39, 27.15, 22.42. ESI-MS $\left(\mathrm{C}_{15} \mathrm{H}_{16} \mathrm{OS}\right): 243[\mathrm{M}-\mathrm{H}]^{-}$.

2-Isopropyl-4-(3-(trifluoromethyl)phenylthio)phenol (3aa). Colorless viscous liquid. ${ }^{1} \mathrm{H}$ NMR (500 $\left.\mathrm{MHz}, \mathrm{CDCl}_{3}, \delta \mathrm{ppm}\right)$ : 7.36-7.30 (m, 3H, ArH), 7.26-7.21 (m, 3H, ArH), 6.79 (d, $J=$ $8.0 \mathrm{~Hz}, 1 \mathrm{H}, \mathrm{ArH}), 5.02(\mathrm{~s}, 1 \mathrm{H}, \mathrm{OH}), 3.23-3.16(\mathrm{~m}, 1 \mathrm{H}, \mathrm{CH}), 1.25$ $\left(\mathrm{d}, J=7.0 \mathrm{~Hz}, 6 \mathrm{H}, 2 \times \mathrm{CH}_{3}\right) .{ }^{13} \mathrm{C} \mathrm{NMR}\left(125 \mathrm{MHz}, \mathrm{CDCl}_{3}, \delta \mathrm{ppm}\right)$ : 153.82, 141.09, 136.31, 133.37, 133.31, 131.39, 130.07, 129.15, $124.93,123.52,123.49,122.36,121.96,116.68,27.12$, 22.38. ESIMS $\left(\mathrm{C}_{16} \mathrm{H}_{15} \mathrm{~F}_{3} \mathrm{OS}\right): 311[\mathrm{M}-\mathrm{H}]^{-}$.

2-Isopropyl-4-(p-tolylthio)phenol (3ab). Brown viscous liquid. ${ }^{1} \mathrm{H}$ NMR (500 MHz, $\left.\mathrm{CDCl}_{3}, \delta \mathrm{ppm}\right): 7.30$ (d, $\left.J=2.0 \mathrm{~Hz}, 1 \mathrm{H}, \mathrm{ArH}\right)$, 7.13-7.10 (m, 3H, ArH), 7.06 (d, $J=8.0 \mathrm{~Hz}, 2 \mathrm{H}, \mathrm{ArH}), 6.71$ (d, $J=$ $8.0 \mathrm{~Hz}, 1 \mathrm{H}, \mathrm{ArH}), 4.92$ (s, 1H, OH), 3.20-3.14 (m, 1H, CH), 2.29 $\left(\mathrm{s}, 3 \mathrm{H}, \mathrm{CH}_{3}\right), 1.23\left(\mathrm{~d}, J=6.5 \mathrm{~Hz}, 6 \mathrm{H}, 2 \times \mathrm{CH}_{3}\right) \cdot{ }^{13} \mathrm{C}$ NMR $(125$ $\left.\mathrm{MHz}, \mathrm{CDCl}_{3}, \delta \mathrm{ppm}\right): 152.83,135.88,135.63,134.60,131.72$, $131.70,129.71,128.95,125.44,116.29,27.16,22.41$, 20.96. EIMS $\left(\mathrm{C}_{16} \mathrm{H}_{18} \mathrm{OS}\right): 258[\mathrm{M}], 259[\mathrm{M}+\mathrm{H}]^{+}$.

4-(p-Tolylthio)aniline (3ac). Brown solid. Mp: 70-71 ${ }^{\circ} \mathrm{C} .{ }^{1} \mathrm{H}$ NMR (500 MHz, $\mathrm{CDCl}_{3}, \delta \mathrm{ppm}$ ): 7.29-7.26 (m, 2H, ArH), 7.107.03 (m, 4H, ArH), 6.67-6.64 (m, 2H, ArH), 3.76 (br s, 2H, $\mathrm{NH}_{2}$ ), $2.29\left(\mathrm{~s}, 3 \mathrm{H}, \mathrm{CH}_{3}\right) .{ }^{13} \mathrm{C} \mathrm{NMR}\left(125 \mathrm{MHz}, \mathrm{CDCl}_{3}, \delta \mathrm{ppm}\right): 146.65$, 135.56, 135.47, 135.33, 129.64, 128.31, 121.79, 115.84, 20.94 EIMS ( $\left.\mathrm{C}_{13} \mathrm{H}_{13} \mathrm{NS}\right): 215[\mathrm{M}], 216[\mathrm{M}+\mathrm{H}]^{+}$.

4-(Phenylthio)aniline (3ad). White solid. Mp: 92-93 ${ }^{\circ} \mathrm{C} .{ }^{1} \mathrm{H}$ NMR (500 MHz, $\left.\mathrm{CDCl}_{3}, \delta \mathrm{ppm}\right): 7.32-7.30$ (m, 2H, ArH), 7.22$7.19(\mathrm{~m}, 2 \mathrm{H}, \mathrm{ArH}), 7.13-7.08$ (m, 3H, ArH), 6.69 (dd, $J=2.0$, $8.5 \mathrm{~Hz}, 2 \mathrm{H}, \mathrm{ArH}$ ), 3.83 (br s, $2 \mathrm{H}, \mathrm{NH}_{2}$ ). ${ }^{13} \mathrm{C}$ NMR $(125 \mathrm{MHz}$, $\left.\mathrm{CDCl}_{3}, \delta \mathrm{ppm}\right): 146.99,139.66,136.09,128.77,127.26,125.21$, 120.48, 115.84. EI-MS ( $\left.\mathrm{C}_{12} \mathrm{H}_{11} \mathrm{NS}\right): 201[\mathrm{M}+\mathrm{H}]^{+}$.

4-(4-Nitrophenylthio)aniline (3ae). Yellow solid. Mp: 137$138{ }^{\circ} \mathrm{C} .{ }^{1} \mathrm{H}$ NMR $\left(500 \mathrm{MHz}, \mathrm{CDCl}_{3}, \delta \mathrm{ppm}\right): 8.03(\mathrm{~d}, J=9.0 \mathrm{~Hz}$, $2 \mathrm{H}, \mathrm{ArH}), 7.34$ (d, $J=8.5 \mathrm{~Hz}, 2 \mathrm{H}, \operatorname{ArH}), 7.09$ (d, $J=9.0 \mathrm{~Hz}, 2 \mathrm{H}$, ArH), 6.74 (d, $J=8.5 \mathrm{~Hz}, 2 \mathrm{H}, \mathrm{ArH}), 3.96$ (br s, $\left.2 \mathrm{H}, \mathrm{NH}_{2}\right) \cdot{ }^{13} \mathrm{C} \mathrm{NMR}$ (125 MHz, $\left.\mathrm{CDCl}_{3}, \delta \mathrm{ppm}\right): 151.04,148.36,144.83,137.24$, 125.24, 123.89, 116.45, 116.15. EI-MS $\left(\mathrm{C}_{12} \mathrm{H}_{10} \mathrm{~N}_{2} \mathrm{O}_{2} \mathrm{~S}\right): 246$ [M], $247[\mathrm{M}+\mathrm{H}]^{+}$.

2-Methyl-4-(phenylthio)aniline (3af). Brown solid. Mp: 59$60{ }^{\circ} \mathrm{C} .{ }^{1} \mathrm{H}$ NMR $\left(500 \mathrm{MHz}, \mathrm{CDCl}_{3}, \delta \mathrm{ppm}\right): 7.25-7.19(\mathrm{~m}, 4 \mathrm{H}$, ArH), 7.13-7.08 (m, 3H, ArH), 6.68 (dd, $J=8.0 \mathrm{~Hz}, 1 \mathrm{H}, \mathrm{ArH}), 3.78$ (br s, $2 \mathrm{H}, \mathrm{NH}_{2}$ ), 2.15 (s, 3H, $\left.\mathrm{CH}_{3}\right) .{ }^{13} \mathrm{C} \mathrm{NMR} \mathrm{(125} \mathrm{MHz}, \mathrm{CDCl}_{3}$, $\delta$ ppm): 145.35, 139.94, 137.02, 133.88, 128.81, 128.78, 127.18, $125.14,123.29,120.17,115.87,17.23$. ESI-MS ( $\left.\mathrm{C}_{13} \mathrm{H}_{13} \mathrm{NS}\right): 216$ [M $+\mathrm{H}]^{+}$.

2-Methyl-4-(4-nitrophenylthio)aniline (3ag). Yellow solid. Mp: 121-122 ${ }^{\circ} \mathrm{C} .{ }^{1} \mathrm{H}$ NMR (500 MHz, $\left.\mathrm{CDCl}_{3}, \delta \mathrm{ppm}\right): 8.03$ (dd, $J=2.0$, $7.0 \mathrm{~Hz}, 2 \mathrm{H}, \mathrm{ArH}), 7.26-7.21(\mathrm{~m}, 2 \mathrm{H}, \operatorname{ArH}), 7.09$ (dd, $J=2.0$, $7.0 \mathrm{~Hz}, 2 \mathrm{H}, \mathrm{ArH}), 6.74$ (d, $J=8.0 \mathrm{~Hz}, 1 \mathrm{H}, \mathrm{ArH}) 3.89$ (br s, 2H, $\mathrm{NH}_{2}$ ), $2.18\left(\mathrm{~s}, 3 \mathrm{H}, \mathrm{CH}_{3}\right) .{ }^{13} \mathrm{C} \mathrm{NMR}\left(125 \mathrm{MHz}, \mathrm{CDCl}_{3}, \delta \mathrm{ppm}\right)$ : 151.24, 146.61, 144.78, 137.88, 134.88, 125.23, 123.87, 123.70, 116.28, 115.87, 17.23. ESI-MS $\left(\mathrm{C}_{13} \mathrm{H}_{12} \mathrm{~N}_{2} \mathrm{O}_{2} \mathrm{~S}\right): 261[\mathrm{M}+\mathrm{H}]^{+}$. 
3-(Phenylthio)-1H-indole (5a). White solid. Mp: $150-151{ }^{\circ} \mathrm{C} .{ }^{1} \mathrm{H}$ NMR (500 MHz, $\left.\mathrm{CDCl}_{3}, \delta \mathrm{ppm}\right): 8.41$ (br s, 1H, NH), 7.62 (d, $J=$ $8.0 \mathrm{~Hz}, 1 \mathrm{H}, \operatorname{ArH}), 7.50$ (d, $J=2.5 \mathrm{~Hz}, 1 \mathrm{H}, \operatorname{ArH}), 7.45$ (d, $J=$ $8.5 \mathrm{~Hz}, 1 \mathrm{H}, \mathrm{ArH}), 7.29-7.26(\mathrm{~m}, 1 \mathrm{H}, \mathrm{ArH}), 7.18-7.03(\mathrm{~m}, 6 \mathrm{H}$, $\mathrm{ArH}) .{ }^{13} \mathrm{C}$ NMR (125 MHz, $\left.\mathrm{CDCl}_{3}, \delta \mathrm{ppm}\right):$ 139.22, 136.51, 130.64, 129.13, 128.69, 125.87, 124.77, 123.07, 120.92, 119.70, 111.55, 102.95. EI-MS $\left(\mathrm{C}_{14} \mathrm{H}_{11} \mathrm{NS}\right): 226[\mathrm{M}+\mathrm{H}]^{+}$.

3-(3-(Trifluoromethyl)phenylthio)-1H-indole (5b). Brown viscous liquid. ${ }^{1} \mathrm{H} \mathrm{NMR}\left(500 \mathrm{MHz}, \mathrm{CDCl}_{3}, \delta \mathrm{ppm}\right): 8.48$ (br s, $1 \mathrm{H}$, $\mathrm{NH}), 7.63$ (dd, $J=1.0,7.5 \mathrm{~Hz}, 1 \mathrm{H}, \mathrm{ArH}), 7.54$ (d, $J=3.0 \mathrm{~Hz}, 1 \mathrm{H}$, ArH), 7.49 (s, 1H, ArH), 7.48 (s, 1H, ArH), 7.44 (s, 1H, ArH), 7.347.31 (m, 2H, ArH), 7.28-7.21 (m, 2H, ArH). ${ }^{13} \mathrm{C}$ NMR (125 MHz, $\left.\mathrm{CDCl}_{3}, \delta \mathrm{ppm}\right): 141.0,136.56,131.01,129.06(\mathrm{q}), 123.34,122.36$, $122.33,121.53,121.50,121.20,119.41,111.76$, 101.59. EI-MS $\left(\mathrm{C}_{15} \mathrm{H}_{10} \mathrm{~F}_{3} \mathrm{NS}\right): 294[\mathrm{M}+\mathrm{H}]^{+}$.

3-(4-Nitrophenylthio)-1H-indole (5c). Brown solid. Mp: 176$177{ }^{\circ} \mathrm{C} .{ }^{1} \mathrm{H}$ NMR $\left(500 \mathrm{MHz}, \mathrm{CDCl}_{3}, \delta \mathrm{ppm}\right): 8.63$ (br s, $\left.1 \mathrm{H}, \mathrm{NH}\right)$, 8.01-7.98 (m, 2H, ArH), 7.55-7.49 (m, 3H, ArH), 7.33-7.26 (m, 1H, ArH), 7.21-7.11 (m, 3H, ArH). ${ }^{13} \mathrm{C}$ NMR (125 MHz, $\mathrm{CDCl}_{3}$, $\delta$ ppm): 149.82, 144.93, 136.61, 131.21, 128.45, 125.11, 123.88, $123.58,121.44,119.23,111.97,100.23$. EI-MS $\left(\mathrm{C}_{14} \mathrm{H}_{10} \mathrm{~N}_{2} \mathrm{O}_{2} \mathrm{~S}\right)$ : $270[\mathrm{M}], 271[\mathrm{M}+\mathrm{H}]^{+}$.

3-(p-Tolylthio)-1H-indole (5d). White solid. Mp: $123-124{ }^{\circ} \mathrm{C}$. ${ }^{1} \mathrm{H}$ NMR (500 MHz, $\left.\mathrm{CDCl}_{3}, \delta \mathrm{ppm}\right): 8.36$ (br s, $\left.1 \mathrm{H}, \mathrm{NH}\right), 7.62$ (d, $=8.0 \mathrm{~Hz}, 1 \mathrm{H}, \mathrm{ArH}), 7.48(\mathrm{~d}, J=2.5 \mathrm{~Hz}, 1 \mathrm{H}, \operatorname{ArH}), 7.44(\mathrm{~d}, J=$ $8.0 \mathrm{~Hz}, 1 \mathrm{H}, \mathrm{ArH}), 7.27-7.24(\mathrm{~m}, 1 \mathrm{H}, \mathrm{ArH}), 7.17-7.14(\mathrm{~m}, 1 \mathrm{H}$, ArH), 7.04 (dd, $J=2.0,7.0 \mathrm{~Hz}, 2 \mathrm{H}, \mathrm{ArH}), 6.98$ (d, $J=8.0 \mathrm{~Hz}, 2 \mathrm{H}$, $\mathrm{ArH}), 2.25$ (s, 3H, $\left.\mathrm{CH}_{3}\right) .{ }^{13} \mathrm{C} \mathrm{NMR}\left(125 \mathrm{MHz}, \mathrm{CDCl}_{3}, \delta \mathrm{ppm}\right)$ : 136.48, 135.48, 134.65, 130.38, 129.48, 129.14, 126.29, 122.99, 120.84, 119.73, 111.51, 103.62, 20.86. EI-MS ( $\left.\mathrm{C}_{15} \mathrm{H}_{13} \mathrm{NS}\right): 239$ $[\mathrm{M}], 240[\mathrm{M}+\mathrm{H}]^{+}$.

1-(Phenylthio)naphthalen-2-ol (7a). White solid. Mp: 52-53 ${ }^{\circ} \mathrm{C}$. ${ }^{1} \mathrm{H}$ NMR (500 MHz, $\left.\mathrm{CDCl}_{3}, \delta \mathrm{ppm}\right): 8.24$ (d, $\left.J=8.0 \mathrm{~Hz}, 1 \mathrm{H}, \operatorname{ArH}\right)$, 7.93 (d, $J=9.0 \mathrm{~Hz}, 1 \mathrm{H}, \mathrm{ArH}), 7.83$ (d, $J=8.0 \mathrm{~Hz}, 1 \mathrm{H}, \mathrm{ArH}), 7.52-$ 7.49 (m, 1H, ArH), 7.40-7.31 (m, 2H, ArH), 7.20-7.16 (m, 2H, ArH), 7.13-7.10 (m, 1H, ArH), 7.05-7.03 (m, 2H, ArH). ${ }^{13} \mathrm{C}$ NMR (125 MHz, $\left.\mathrm{CDCl}_{3}, \delta \mathrm{ppm}\right): 157.03,135.47,135.39,132.86$, 129.52, 129.21, 128.60, 127.98, 126.40, 125.92, 124.72, 123.89, 116.90, 108.07. EI-MS $\left(\mathrm{C}_{16} \mathrm{H}_{12} \mathrm{OS}\right): 252[\mathrm{M}], 253[\mathrm{M}+\mathrm{H}]^{+}$.

1-(4-tert-Butylphenylthio)naphthalen-2-ol (7b). White solid. Mp: 71-72 ${ }^{\circ} \mathrm{C} .{ }^{1} \mathrm{H}$ NMR (500 $\left.\mathrm{MHz}, \mathrm{CDCl}_{3}, \delta \mathrm{ppm}\right): 8.28$ (d, $J=$ $8.0 \mathrm{~Hz}, 1 \mathrm{H}, \operatorname{ArH}), 7.91$ (d, $J=9.0 \mathrm{~Hz}, 1 \mathrm{H}, \operatorname{ArH}), 7.83$ (d, $J=$ $8.5 \mathrm{~Hz}, 1 \mathrm{H}, \mathrm{ArH}), 7.52-7.49(\mathrm{~m}, 1 \mathrm{H}, \mathrm{ArH}), 7.39-7.34(\mathrm{~m}, 2 \mathrm{H}$, ArH), 7.23-7.19 (m, 3H, ArH, OH), 7.00-6.97 (m, 2H, ArH), 1.24 $\left(\mathrm{s}, 9 \mathrm{H}, 3 \times \mathrm{CH}_{3}\right) \cdot{ }^{13} \mathrm{C} \mathrm{NMR}\left(125 \mathrm{MHz}, \mathrm{CDCl}_{3}, \delta \mathrm{ppm}\right): 156.92$, $149.14,135.57,132.68,131.81,129.48,128.55,127.89,127.78$, 126.30, 126.28, 126.15, 124.84, 123.82, 116.85, 108.57, 34.39, 31.29, 31.23. EI-MS $\left(\mathrm{C}_{20} \mathrm{H}_{20} \mathrm{OS}\right): 308[\mathrm{M}], 309[\mathrm{M}+\mathrm{H}]^{+}$.

1-(p-Tolylthio)naphthalen-2-ol (7c). White solid. Mp: 80-81 ${ }^{\circ} \mathrm{C}$. ${ }^{1} \mathrm{H}$ NMR (500 MHz, $\left.\mathrm{CDCl}_{3}, \delta \mathrm{ppm}\right): 8.24$ (d, $\left.J=8.5 \mathrm{~Hz}, 1 \mathrm{H}, \mathrm{ArH}\right)$, $7.90(\mathrm{~d}, J=9.0 \mathrm{~Hz}, 1 \mathrm{H}, \mathrm{ArH}), 7.82$ (d, $J=8.0 \mathrm{~Hz}, 1 \mathrm{H}, \mathrm{ArH}), 7.51-$ 7.47 (m, 1H, ArH), 7.38-7.32 (m, 2H, ArH), 7.20 (br s, $1 \mathrm{H}, \mathrm{OH}$ ), 7.0-6.94 (m, 4H, ArH), 2.24 (s, 3H, $\left.\mathrm{CH}_{3}\right) .{ }^{13} \mathrm{C}$ NMR $(125 \mathrm{MHz}$, $\left.\mathrm{CDCl}_{3}, \delta \mathrm{ppm}\right): 156.88,135.90,135.45,132.65,131.75,129.96$, $129.49,128.55,127.89,126.70,124.75,123.80,116.84,108.71$, 20.89. EI-MS $\left(\mathrm{C}_{17} \mathrm{H}_{14} \mathrm{OS}\right): 266[\mathrm{M}], 267[\mathrm{M}+\mathrm{H}]^{+}$.
6-Methoxy-1-(4-nitrophenylthio)-1,2,3,4-tetrahydronaphthalen1-ol (9a). Yellow solid. Mp: 191-192 ${ }^{\circ} \mathrm{C} .{ }^{1} \mathrm{H}$ NMR $(500 \mathrm{MHz}$, $\left.\mathrm{CDCl}_{3}, \delta \mathrm{ppm}\right): 8.38-8.35$ (m, 2H, ArH), 8.24-8.21 (m, 2H, ArH), $7.90(\mathrm{~d}, J=9.0 \mathrm{~Hz}, 1 \mathrm{H}, \mathrm{ArH}), 7.47$ (br s, 1H, OH), 6.78 (dd, $J=$ 2.5, 9.0 Hz, 1H, ArH), 6.60 (d, $J=2.5 \mathrm{~Hz}, 1 \mathrm{H}, \operatorname{ArH}), 3.80$ (s, 3H, $\left.\mathrm{OCH}_{3}\right), 2.71\left(\mathrm{t}, J=6.0 \mathrm{~Hz}, 2 \mathrm{H}, \mathrm{CH}_{2}\right), 2.46\left(\mathrm{t}, J=6.5 \mathrm{~Hz}, 2 \mathrm{H}, \mathrm{CH}_{2}\right)$, 1.92-1.87 (m, 2H, $\left.\mathrm{CH}_{2}\right) .{ }^{13} \mathrm{C}$ NMR (125 MHz, $\left.\mathrm{CDCl}_{3}, \delta \mathrm{ppm}\right)$ : $161.06,154.29,150.39$, 144.10, 141.85, 129.51, 126.68, 124.13, 123.88, 113.29, 112.67, 55.31, 29.71, 25.44, 21.41. EI-MS $\left(\mathrm{C}_{17} \mathrm{H}_{17} \mathrm{~N}_{4} \mathrm{OS}\right): 331[\mathrm{M}], 332[\mathrm{M}+\mathrm{H}]^{+}$.

4-(4-Nitrophenylthio)-2-phenylchroman-4-ol (9b). White solid. Mp: $171-172{ }^{\circ} \mathrm{C} .{ }^{1} \mathrm{H}$ NMR (500 $\left.\mathrm{MHz}, \mathrm{CDCl}_{3}, \delta \mathrm{ppm}\right): 8.36$ (dd, $J=$ 2.0, 7.0 Hz, 2H, ArH), 8.20 (dd, $J=2.0,7.0 \mathrm{~Hz}, 2 \mathrm{H}, \mathrm{ArH}), 7.89$ (dd, $J$ $=1.5,8.0 \mathrm{~Hz}, 1 \mathrm{H}, \mathrm{ArH}), 7.63(\mathrm{br}, 1 \mathrm{H}, \mathrm{OH}), 7.43-7.30(\mathrm{~m}, 6 \mathrm{H}$, ArH), 7.02-6.94 (m, 2H, ArH), 5.10-5.07 (m, 1H, CH), 3.04-3.00 (m, $1 \mathrm{H}, \mathrm{CH}), 2.65 .04-2.59(\mathrm{~m}, 1 \mathrm{H}, \mathrm{CH}) .{ }^{13} \mathrm{C} \mathrm{NMR}\left(125 \mathrm{MHz}, \mathrm{CDCl}_{3}\right.$, $\delta$ ppm): 157.41, 150.50, 150.06, 143.68, 138.94, 132.46, 129.50, $128.90,128.86,126.04,124.67,124.23,122.02,118.79,118.08$, 76.79, 32.50. ESI-MS $\left(\mathrm{C}_{21} \mathrm{H}_{17} \mathrm{NO}_{4} \mathrm{~S}\right): 379[\mathrm{M}], 380[\mathrm{M}+\mathrm{H}]^{+}$.

\section{Acknowledgements}

The authors are thankful to the Department of Science and Technology for DST-INSPIRE Faculty awards (IFA 14-CH-145) and Director CSIR-CIMAP for providing necessary facilities.

\section{Notes and references}

1 (a) C. Shen, P. Zhang, Q. Sun, S. Bai, T. S. A. Hor and X. Liu, Chem. Soc. Rev., 2015, 44, 291-314; (b) T. Mori, T. Nishimura, T. Yamamoto, I. Doi, E. Miyazaki, I. Osaka and K. Takimiya, J. Am. Chem. Soc., 2013, 135, 13900-13913; (c) M. Gingras, Y. M. Chabre, M. Roy and R. Roy, Chem. Soc. Rev., 2013, 42, 4823-4841; (d) H. Liu and X. Jiang, Chem.-Asian J., 2013, 8, 2546-2563; (e) K. Takimiya, S. Shinamura, I. Osaka and E. Miyazaki, Adv. Mater., 2011, 23, 4347-4370; (f) T. Nakazawa, J. Xu, T. Nishikawa, T. Oda, A. Fujita, K. Ukai, R. E. P. Mangindaan, H. Rotinsulu, H. Kobayashi and M. Namikoshi, J. Nat. Prod., 2007, 70, 439-442; (g) T. Oda, T. Fujiwara, H. Liu, K. Ukai, R. E. P. Mangindaan, M. Mochizuki and M. Namikoshi, Mar. Drugs, 2006, 4, 1521; (h) A. Y. Sizov, A. N. Kovergin and A. F. Ermolov, Russ. Chem. Rev., 2003, 72, 357-374; (i) I. P. Beletskaya and V. P. Ananikov, Chem. Rev., 2011, 111, 1596-1636; (j) T. Kondo and T.-A. Mitsudo, Chem. Rev., 2000, 100, 32053220 .

2 (a) J. Hassan, M. Sevignon, C. Gozzi, E. Schulz and M. Lemaire, Chem. Rev., 2002, 102, 1359-1470; (b) S. V. Ley and A. W. Thoma, Angew. Chem., Int. Ed., 2003, 42, 54005449; (c) S.-R. Guo, Y.-Q. Yuan and J.-N. Xiang, Org. Lett., 2013, 15, 4654-4657.

3 T. Kondo and T. Mitsudo, Chem. Rev., 2000, 100, 3205-3220. 4 (a) Y. Zhang, K. N. Ngeow and J. Y. Ying, Org. Lett., 2007, 9, 3495-3499; (b) A. Saxena, A. Kumar and S. Mozumdar, Appl. Catal., A, 2007, 317, 210-217; (c) D. Alves, R. G. Lara, M. E. Contreira, C. S. Radatz, L. F. B. Duarte and G. Perin, Tetrahedron Lett., 2012, 53, 3364-3368; (d) V. P. Reddy, 
K. Swapna, A. V. Kumar and K. R. Rao, J. Org. Chem., 2009, 74, 3189-3191; (e) X. Chen, S.-S. Hao, C. E. Goodhue and J.-Q. Yu, J. Am. Chem. Soc., 2006, 128, 6790-6791; (f) L.-H. Zou, D. Priebbenow, L. Wang, J. Mottweiler and C. Bolm, Adv. Synth. Catal., 2013, 355, 2558-2563; (g) F.-L. Yang, X.-T. Ma and S.-K. Tian, Chem.-Eur. J., 2012, 18, 1582-1585; (h) Y. Li, J. Pu and X. Jiang, Org. Lett., 2014, 16, 2692-2695; (i) T.-T. Wang, F.-X. Wang, F.-L. Yang and S.-K. Tian, Chem. Commun., 2014, 50, 3802-3805; (j) S.-R. Guo, Y.-Q. Yuan and J.-N. Xiang, Org. Lett., 2013, 15, 4654-4657; (k) X. Yu, X. Li and B. Wan, Org. Biomol. Chem., 2012, 10, 7429-7438.

5 (a) W. Wei, C. Liu, D. Yang, J. Wen, J. You, Y. Suo and H. Wang, Chem. Commun., 2013, 49, 10239-10241; (b) S. Zhang, P. Qian, M. Zhang, M. Hu and J. Cheng, J. Org. Chem., 2010, 75, 6732-6735; (c) X. Li, Y. Xu, W. Wu, C. Jiang, C. Qi and H. Jiang, Chem.-Eur. J., 2014, 20, 79117915.

6 (a) H. Tian, C. Zhu, H. Yang and H. Fu, Chem. Commun., 2014, 50, 8875-8877; (b) C. Lin, D. Li, B. Wang, J. Yao and Y. Zhang, Org. Lett., 2015, 17, 1328-1331; (c) C. Lin, W. Yu, J. Yao, B. Wang, Z. Liu and Y. Zhang, Org. Lett., 2015, 17, 1340-1343.

7 (a) Y. Yang, W. Hou, L. Qin, J. Du, H. Feng, B. Zhou and Y. Li, Chem.-Eur. J., 2014, 20, 416-420; (b) Z. Chen, B. Wang, J. Zhang, W. Yu, Z. Liu and Y. Zhang, Org. Chem. Front., 2015, 2, 1107-1295.

8 (a) B. Liu, J. Li, F. Song and J. You, Chem.-Eur. J., 2012, 18, 10830-10833; (b) S.-R. Guo, W.-M. He, J.-N. Xiang and Y.-Q. Yuan, Chem. Commun., 2014, 50, 8578-8581; (c) P. Saravanan and P. Anbarasan, Org. Lett., 2014, 16, 848851; (d) M. Iwasaki, M. Iyanaga, Y. Tsuchiya, Y. Nishimura, W. Li, Z. Li and Y. Nishihara, Chem.-Eur. J., 2014, 20, 2459-2462.

9 (a) F. Xiao, S. Chen, J. Tian, H. Huang, Y. Liu and G.-J. Deng, Green Chem., 2016, 18, 1538-1546; (b) X. Kang, R. Yan, G. Yu, X. Pang, X. Liu, X. Li, L. Xiang and G. Huang, J. Org. Chem., 2014, 79, 10605-10610.

10 (a) D. Wang, S. Guo, R. Zhang, S. Lin and Z. Yan, RSC Adv., 2016, 6, 54377-54381; (b) A. Hajra, A. K. Bagdi, S. Mitra and M. Ghosh, Org. Biomol. Chem., 2015, 13, 3314-3320.

11 Y. Xu, X. Tang, W. Hu, W. Wu and H. Jiang, Green Chem., 2014, 16, 3720-3723.

12 (a) F.-L. Yang, F.-X. Wang, T.-T. Wang, Y.-J. Wang and S.-K. Tian, Chem. Commun., 2014, 50, 2111-2113; (b) G. Kumaraswamy, R. Raju and V. Narayanarao, RSC Adv., 2015, 5, 22718-22723.

13 (a) F. Xiao, H. Xie, S. Liu and G. Deng, Adv. Synth. Catal., 2014, 356, 364-368; (b) R.-Y. Tang, Y.-X. Xie, Y.-L. Xie, J.-N. Xiang and J.-H. Li, Chem. Commun., 2011, 47, 1286712869.
14 (a) S. Chowdhury, R. S. Mohan and J. L. Scott, Tetrahedron, 2007, 63, 2363-2389; (b) Q. Zhang, S. Zhang and Y. Deng, Green Chem., 2011, 13, 2619-2637.

15 (a) W. Bao and Z. Wang, Green Chem., 2006, 8, 1028-1033; (b) M. A. P. Martins, C. P. Frizzo, D. N. Moreira, N. Zanatta and H. G. Bonacorso, Chem. Rev., 2008, 108, 2015-2050.

16 D. Zhao, M. Wu, Y. Kou and E. Min, Catal. Today, 2002, 74, 157-189.

17 (a) J. Dupont, R. F. de Souza and P. A. Z. Suarez, Chem. Rev., 2002, 102, 3667-3691; (b) D. S. Raghuvanshi and K. N. Singh, Tetrahedron Lett., 2011, 52, 5702-5705.

18 (a) K. Qiao and C. Yakoyama, Chem. Lett., 2004, 33, 472-473; (b) J. P. Hallett and T. Welton, Chem. Rev., 2011, 111, 35083576.

19 (a) V. I. Pârvulescu and C. Hardacre, Chem. Rev., 2007, 107, 2615-2665; (b) W. Sun, C.-G. Xia and H.-W. Wang, Tetrahedron Lett., 2003, 44, 2409-2411.

20 (a) A. Kamal and G. Chouhan, Tetrahedron Lett., 2005, 46, 1489-1491; (b) M. J. Earle, S. P. Katdare and K. R. Seddon, Org. Lett., 2004, 6, 707-710.

21 (a) N. Singh, R. Singh, D. S. Raghuvanshi and K. N. Singh, Org. Lett., 2013, 15, 5874-5877; (b) N. Singh, B. K. Allam, D. S. Raghuvanshi and K. N. Singh, Adv. Synth. Catal., 2013, 355, 1840-1848; (c) R. Singh, D. S. Raghuvanshi and K. N. Singh, Org. Lett., 2013, 15, 4202-4205; (d) D. P. Singh, D. S. Raghuvanshi, K. N. Singh and V. P. Singh, J. Mol. Catal. A: Chem., 2013, 379, 21-29; (e) D. S. Raghuvanshi, A. K. Gupta and K. N. Singh, Org. Lett., 2012, 14, 4326-4329. 22 (a) F.-L. Yang, X.-T. Ma and S.-K. Tian, Chem.-Eur. J., 2012, 18, 1582-1585; (b) B. Liu, J. Li, F. Song and J. You, Chem.Eur. J., 2012, 18, 10830-10833; (c) X. Yu, X. Li and B. Wan, Org. Biomol. Chem., 2012, 10, 7479-7482; (d) O. Y. Yuen, C. M. So, W. T. Wong and F. Y. Kwong, Synlett, 2012, 23, 2714-2718.

23 (a) X. Li, X. Xu and C. Zhou, Chem. Commun., 2012, 48, 12240-12242; (b) T. Taniguchi, A. Idota and H. Ishibashi, Org. Biomol. Chem., 2011, 9, 3151-3153; (c) I. V. Ukrainets, A. A. Tkach, V. V. Kravtsova and S. V. Shishkina, Chem. Heterocycl. Compd., 2008, 44, 677-681; (d) S. Kamijo, M. AlMasum and Y. Yamamoto, Tetrahedron Lett., 1998, 39, 691695; (e) R. Ballini, E. Marcantoni and M. Petrin, Tetrahedron, 1989, 45, 6791-6798.

24 (a) D. J. Pasto and R. T. Taylor, Org. React., 1991, 40, 91-155; (b) D. J. Pasto, in Comprehensive Organic Synthesis, ed. B. M. Trost and I. Fleming, Pergamon Press, Oxford, 1991, vol. 8, pp. 471-488.

25 W. Ge, X. Zhu and Y. Wei, Adv. Synth. Catal., 2013, 355, 30143021. 